\title{
Magnetically charged calorons with non-trivial holonomy
}

\author{
Takumi Kato, Atsushi Nakamula ${ }^{1}$ and Koki Takesue \\ Department of Physics, School of Science, Kitasato University, \\ Sagamihara 252-0373, Japan \\ E-mail: tkato@sci.kitasato-u.ac.jp, nakamula@sci.kitasato-u.ac.jp, \\ ktakesue@sci.kitasato-u.ac.jp
}

ABSTRACT: Instantons in pure Yang-Mills theories on partially periodic space $\mathbb{R}^{3} \times S^{1}$ are usually called calorons. The background periodicity brings on characteristic features of calorons such as non-trivial holonomy, which plays an essential role for confinement/deconfinement transition in pure Yang-Mills gauge theory. For the case of gauge group $\mathrm{SU}(2)$, calorons can be interpreted as composite objects of two constituent "monopoles" with opposite magnetic charges. There are often the cases that the two monopole charges are unbalanced so that the calorons possess net magnetic charge in $\mathbb{R}^{3}$. In this paper, we consider several mechanism how such net magnetic charges appear for certain types of calorons through the ADHM/Nahm construction with explicit examples. In particular, we construct analytically the gauge configuration of the $(2,1)$-caloron with $\mathrm{U}(1)$-symmetry, which has intrinsically magnetic charge.

KEywords: Solitons Monopoles and Instantons, Integrable Field Theories, Wilson, 't Hooft and Polyakov loops

ARXIV EPRINT: 1804.03268

\footnotetext{
${ }^{1}$ Corresponding author.
} 


\section{Contents}

1 Introduction 1

2 Large scale limits and magnetic charges 3

2.1 Jackiw-Nohl-Rebbi calorons 4

2.2 KvBLL calorons 5

2.3 (2,2)-calorons 6

3 Intrinsically charged calorons $\quad 9$

$\begin{array}{lll}3.1 & (2,1) \text {-calorons } & 10\end{array}$

$\begin{array}{lll}3.1 .1 & \text { ADHM/Nahm construction for }\left(N, N^{\prime}\right) \text {-calorons } & 10\end{array}$

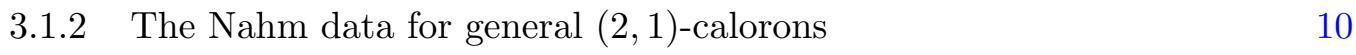

3.2 Nahm transform for $(2,1)$-calorons with U(1)-symmetry 11

4 Decomposable Nahm data and removing limits $\quad 17$

$\begin{array}{lll}4.1 & \text { Nahm data of }(2,1 \oplus 1) \text {-calorons } & 17\end{array}$

4.2 Removing limit to $(2,1)$-caloron Nahm data 20

5 Summary and outlook $\quad 21$

A Harrington-Shepard 2-caloron $\quad 22$

$\begin{array}{ll}\text { B } \operatorname{KvBLL}(1,1) \text {-caloron } & 25\end{array}$

\section{Introduction}

Calorons are topological solitons to the (anti-)self-dual (ASD) Yang-Mills theories on $\mathbb{R}^{3} \times S^{1}$. They have a remarkable feature due to the periodic nature of the background space, namely holonomy around $S^{1}$, which plays a crucial role for confinement/deconfinement phase transition and related non-perturbative effects in finite temperature QCD and QCDlike theories [1-9]. On the one hand, there is an interpretation of calorons as interpolating objects between instantons and monopoles, which interpretation is useful for the understanding of D-branes [10].

Similarly to several topological solitons on periodic backgrounds, calorons can be seen as composite objects of elementary solitons $[11,12]$. For $\mathrm{SU}(\mathcal{N})$ gauge theory, the elementary solitons are $\mathcal{N}$ individual monopoles in $\mathbb{R}^{3}$, which are refered to as "instantondyons" or "instanton-monopoles" in the context of confinement/deconfinement phase transition [13-15]. Due to this fact, calorons are sometimes able to possess net magnetic charges. We should remark that, although the monopoles have translational invariance in the $S^{1}$-direction, the composite objects do not, in general. For example, calorons in SU(2) Yang-Mills theory are composed of two individual monopoles with topological numbers, say, $N$ and $N^{\prime}$. This hybrid structure of SU(2) calorons has been obtained firstly by Kraan 
and van-Baal [16], and independently by Lee and $\mathrm{Lu}$ (KvBLL) [17] for $N=N^{\prime}=1$, usually denoted as $(1,1)$-calorons. Subsequently, the $(2,2)$-calorons have been analysed in [18-20]. Although $N$ and $N^{\prime}$ are both positive integer, the detailed analysis in [17] shows that the constituent monopoles can be interpreted as oppositely charged. Thus, the KvBLL calorons are magnetically neutral, and $\mathrm{SU}(2)$ calorons would have net magnetic charges when $N \neq N^{\prime}$, in general. However, in contrast to the neutrally charged cases, a little systematic analysis is made for the magnetically charged calorons, so far. In fact, as we will show in this papar, the mechanism of the appearance of the net magnetic charges of calorons is not so simple.

It is known that the net magnetic charge of calorons sometimes appears as a "large scale" limit of magnetically neutral calorons. Typical examples of the cases are BogomolnyiPrasad-Sommerfield (BPS) monopole limits [21, 22], which no longer have a holonomy parameter. On the one hand, there exist calorons with proper or intrinsic magnetic charge, i.e., the cases with $N-N^{\prime} \neq 0$. However, only a few example is known for these sort of calorons, except for some particular cases given below. Then a question arises: is there any connection between the magnetically neutral calorons and intrinsically charged ones? So far, however, there has not been carried out extensive research on this direction. In this paper, we consider this subject by focusing on $\mathrm{SU}(2)$ calorons in terms of ADHM/Nahm construction [23-25] with certain examples explicitly. Among them are $(2,1)$-calorons, which are composite objects of oppositely charged monopoles of absolute charge 2 and 1 , so that they have intrinsic magnetic charge 1 . The Nahm data of $(2,1)$-calorons with $\mathrm{U}(1)$ or $\mathrm{SO}(2)$-symmetric type are already introduced by Harland [18]. Here we present the Nahm data of $(2,1)$-calorons without particular symmetry. In addition, we give the analytic gauge fields of the U(1)-symmetric type by Nahm transform. We next consider a novel type of calorons whose constituent monopoles are made of direct sum of two independent 1-monopoles, in order to examine the interrelation between the $(2,1)$-calorons and magnetically neutral calorons. These monopoles are obviously decomposable, and we denote this monopole as type- $(1 \oplus 1)$. Applying this monopole together with another 2monopole, we assemble neutral calorons of type- $(2,1 \oplus 1)$, and take the limit of "1-monopole removing" by decomposing the $(1 \oplus 1)$-monopole. We will show that this removing limit reproduces the charged $(2,1)$-calorons of special moduli parameters. This suggests that not all of the intrinsically charged calorons can be obtained through a limit process from some neutral calorons.

This paper is organized as follows. In section 2, we make a review on the large scale limits and the appearance of the magnetic charges. In section 3 , we consider the intrinsically charged calorons with non-trivial holonomy. As an explicit example, we investigate the Nahm data of $(2,1)$-calorons, and perform the Nahm transform analytically for the U(1)symmetric cases. In section 4 , we consider the interrelation between the neutral calorons of decomposable type and intrinsically charged calorons. In the final section, we summarize the results and make an outlook. 


\section{Large scale limits and magnetic charges}

In this section, we give an overview of the large scale limits and the appearance of magnetic charges of $\mathrm{SU}(2)$ calorons.

Let $\boldsymbol{x}=(x, y, z)$ and $x_{0} \in\left[0,2 \pi / \mu_{0}\right]$ be $\mathbb{R}^{3}$ and $S^{1}$ coordinate, respectively. The magnetic charge and the holonomy are defined from the asymptotic form of the $S^{1}$ or $x_{0}$-component of the gauge potential

$$
\lim _{r \rightarrow \infty} A_{0}(r)=\frac{1}{2}\left(\tilde{\mu}-\frac{Q}{r}\right)(\hat{\boldsymbol{x}} \cdot \boldsymbol{\sigma})+O\left(\frac{1}{r^{2}}\right),
$$

in some gauge, where $r$ is radial coordinate, $\hat{\boldsymbol{x}}$ is unit position vector of $\mathbb{R}^{3}$, and $\tilde{\mu}$ and $Q$ are the holonomy parameter and the magnetic charge, respectively. The Polyakov loop at spatial infinity

$$
\Omega(\infty):=\lim _{r \rightarrow \infty} \mathcal{P} \exp i \int_{S^{1}} A_{0}(x) d x_{0},
$$

is one of the characteristic quantity depending on the holonomy parameter and plays a critical role for the confinement/deconfinement phase transition. If $\Omega(\infty)$ belongs to the center of $\mathrm{SU}(2)$, the holonomy is said to be trivial, whereas maximally non-trivial if $\operatorname{tr} \Omega(\infty)=0$. The holonomy parameter can also be interpreted as the monopole mass from the perspective of monopoles in $\mathbb{R}^{3}$.

We can construct the gauge connection $A_{\mu}$ of calorons through the ADHM/Nahm construction, in which the Nahm data plays an essential role. The Nahm data are given by two parts, the bulk data and the boundary data. The bulk Nahm data, which can be seen as a gauge field on the dual space to the configuration space, are $\mathrm{U}(N)$-valued matrices $T_{\nu}^{(2)}(s)$ and $\mathrm{U}\left(N^{\prime}\right)$-valued one $T_{\nu}^{(1)}(s)(\nu=1,2,3,0)$ defined on one-dimensional interval $s \in I=I_{2} \oplus I_{1}$, respectively. We define $I_{2}=\left(-\mu_{0} / 2,-\mu\right) \oplus\left(\mu, \mu_{0} / 2\right) \simeq\left(\mu, \mu_{0}-\mu\right)$ and $I_{1}=(-\mu, \mu)$, where the periodicity in $s$ is understood. The matrix sizes $N$ on $I_{2}$ and $N^{\prime}$ on $I_{1}$ are interpreted as the absolute values of constituent monopole charges on each interval, and we represent this type of calorons as $\left(N, N^{\prime}\right)$-caloron. Although the matrix sizes $N$ and $N^{\prime}$ are not necessarily identical, we consider the identical cases, i.e., $N=N^{\prime}$, in this section. The nonidentical cases are considered in the following sections, which are the main subjects of this paper. We remark that the order of $N$ and $N^{\prime}$ in the representation $\left(N, N^{\prime}\right)$ is actually not significant, because there exists a large gauge transformation which interchanges the constituents defined on $I_{2}$ and $I_{1}$, called the rotation map [18, 20].

For the gauge connection $A_{\mu}$ to be ASD, the bulk data have to enjoy the Nahm equations

$$
\frac{d T_{j}^{(m)}}{d s}(s)-i\left[T_{0}^{(m)}(s), T_{j}^{(m)}(s)\right]-\frac{i}{2} \epsilon_{j k l}\left[T_{k}^{(m)}(s), T_{l}^{(m)}(s)\right]=0,
$$

where $m=1$ or 2 , and $j, k$ and $l$ take values 1 to 3 . In addition, the reality conditions ${ }^{t} T_{\nu}^{(m)}(s)=T_{\nu}^{(m)}(-s)$ are necessary for the $\mathrm{SU}(2)$ calorons. The boundary data $W$ are 
$N$-row vector of quaternion entries, which enjoy the matching conditions

$$
\begin{aligned}
& \frac{1}{2} \operatorname{Tr} \sigma_{j} W^{\dagger} P_{+} W=T_{j}^{(2)}(\mu)-T_{j}^{(1)}(\mu) \\
& \frac{1}{2} \operatorname{Tr} \sigma_{j} W^{\dagger} P_{-} W=T_{j}^{(1)}(-\mu)-T_{j}^{(2)}\left(\mu_{0}-\mu\right),
\end{aligned}
$$

where $P_{ \pm}=(1 \pm \hat{\boldsymbol{\omega}} \cdot \boldsymbol{\sigma}) / 2$, and $\hat{\boldsymbol{\omega}}$ is a unit vector. In $(2.4 \mathrm{a}),(2.4 \mathrm{~b})$, the traces are taken for quaternions. For the cases with either of $I_{1}$ or $I_{2}$ shrinking to a point, i.e., $\mu \rightarrow 0$ or $\mu \rightarrow \mu_{0} / 2$, one of the constituent monopoles becomes massless. In the cases, say, $\mu \rightarrow \mu_{0} / 2$, the bulk data $T_{j}^{(2)}$ are not necessary, and the matching condition becomes,

$$
T_{j}^{(1)}\left(-\frac{\mu_{0}}{2}\right)-T_{j}^{(1)}\left(\frac{\mu_{0}}{2}\right)=\frac{1}{2} \operatorname{Tr} \sigma_{j} W^{\dagger} W .
$$

We refer to these cases as massless calorons of type $(N,[N])$ (or $([N], N))$, where $[*]$ denotes the massless monopole constituent.

\subsection{Jackiw-Nohl-Rebbi calorons}

The mostly known examples of magnetically charged limits are Jackiw-Nohl-Rebbi (JNR) calorons, which have $Q=1$ and are obtained from Harrington-Shepard (HS) calorons [26, 27]. We briefly make a summary here, for a review of this subject, see [18].

Although the ADHM/Nahm construction can be applied for general cases, the simple way to obtain the HS calorons is given from the 'tHooft ansatz

$$
A_{\mu}=-\frac{1}{2} \eta_{\mu \nu} \partial_{\nu} \log \phi
$$

where $\eta_{\mu \nu}$ is the ASD 'tHooft matrix. We find that the ASD equations are equivalent to the four-dimensional Laplace equation $\partial_{\nu} \partial_{\nu} \phi=0$, which are solved by "point sources" together with a constant term. If the sources, or single instantons, are located at the origin of $\mathbb{R}^{3}$ and aligned periodic on $x_{0}$-axis, we find

$$
\phi=1+\frac{\lambda^{2}}{2 r} \frac{\sinh \mu_{0} r}{\cosh \mu_{0} r-\cos \mu_{0} x_{0}},
$$

where $\lambda$ is the scale of instantons. This is the Harrington-Shepard caloron of instanton number 1 , referred to it as $\mathrm{HS}_{1}$. We can find from direct calculation that asymptotically $A_{0} \sim O\left(1 / r^{2}\right)$, namely $\mathrm{HS}_{1}$ has trivial holonomy and is magnetically neutral. From the $\mathrm{ADHM} / \mathrm{Nahm}$ construction, the Nahm data of $\mathrm{HS}_{1}$ is given by $1 \times 1$ bulk data only on, say $I_{2}$, which is consistent with $\mathrm{HS}_{1}$ being massless. The two constituent monopole charges should be interpreted as opposite sign each other [17], so that the net magnetic charge vanishes. Thus, we can interpret intuitively that the $\mathrm{HS}_{1}$ caloron has constituent monopole of charge $N=1$ on $I_{2}$, and $N^{\prime}=1$ on $I_{1}$ which is massless. We mention here that we ignore the overall sign of the magnetic charges of each constituent, because only the difference $N-N^{\prime}$ is effective throughout this consideration. The standard notation of $\mathrm{HS}_{1}$ caloron is, thus, of the type $(1,[1])$. 
Next, we consider the large scale limit $|\lambda| \rightarrow \infty$ of $\mathrm{HS}_{1}$, which leads to

$$
\phi=\frac{\lambda^{2}}{2 r} \frac{\sinh \mu_{0} r}{\cosh \mu_{0} r-\cos \mu_{0} x_{0}},
$$

this is the JNR caloron of instanton number $1\left(\mathrm{JNR}_{1}\right)$ [28]. In this case, the asymptotic form reads

$$
A_{0}=-\frac{1}{2 r} \hat{\boldsymbol{x}} \cdot \boldsymbol{\sigma}+O\left(\frac{1}{r^{2}}\right),
$$

which shows that $\mathrm{JNR}_{1}$ also has trivial holonomy, but magnetic charge 1, and can be interpreted as $(1,[0])$ caloron. In fact, we can show the gauge equivalence between $\mathrm{JNR}_{1}$ and the BPS 1-monopole $\left(\mathrm{BPS}_{1}\right)[29]$.

This procedure can be generalized to the cases of "instanton number" $N$, i.e., $(N,[N])$ calorons. Namely, the $\mathrm{HS}_{N}$ calorons are given by

$$
\phi=1+\sum_{j=1}^{N} \frac{\lambda_{j}^{2}}{2 r_{j}} \frac{\sinh \mu_{0} r_{j}}{\cosh \mu_{0} r_{j}-\cos \mu_{0} x_{0}},
$$

where $r_{j}=\left|\boldsymbol{x}-\boldsymbol{a}_{j}\right|$ with $\boldsymbol{a}_{j}$ locations of each instanton in $\mathbb{R}^{3}$. Similarly to the $\mathrm{HS}_{1}$, their large scale limits $\lambda_{j} \rightarrow \infty$ lead

$$
\phi=\sum_{j=1}^{N} \frac{\lambda_{j}^{2}}{2 r_{j}} \frac{\sinh \mu_{0} r_{j}}{\cosh \mu_{0} r_{j}-\cos \mu_{0} x_{0}},
$$

which are $\mathrm{JNR}_{N}$ calorons. We can find that the asymptotic forms are equivalent to $\mathrm{JNR}_{1}$,

$$
A_{0}=-\frac{1}{2 r} \hat{\boldsymbol{x}} \cdot \boldsymbol{\sigma}
$$

so that their magnetic charge is 1 . The $\mathrm{JNR}_{N}$ calorons can be interpreted as type $(N,[N-$ $1]$ ), and the $x_{0}$-dependence can not be gauged away except for the $N=1$ case [30]. We remark that further limit from $\mathrm{JNR}_{N}$ to $\mathrm{HS}_{N-1}=(N-1,[N-1])$ is available, i.e., the sequence $\mathrm{HS}_{N} \rightarrow \mathrm{JNR}_{N} \rightarrow \mathrm{HS}_{N-1} \rightarrow \cdots \rightarrow \mathrm{HS}_{1} \rightarrow \mathrm{JNR}_{1} \simeq \mathrm{BPS}_{1}$ holds. For the detail, see [18]. The ADHM/Nahm construction of $\mathrm{HS}_{2}$ and its $\mathrm{JNR}_{2}$ limit is given in detail in appendix A.

\subsection{KvBLL calorons}

Next, we consider the large scale limits of calorons with a holonomy parameter. The simplest example is the case of instanton number 1, i.e., $\operatorname{KvBLL}(1,1)$-calorons $[16,17]$, for which the gauge potential can be constructed analytically from the ADHM/Nahm construction. The Nahm construction to this KvBLL calorons is briefly performed in appendix B.

In this case, the bulk Nahm data is two $1 \times 1$ matrix valued vectors defined on intervals $I_{1}=(-\mu, \mu)$ and $I_{2}=\left(-\mu_{0} / 2,-\mu\right) \oplus\left(\mu, \mu_{0} / 2\right)$, given by

$$
\begin{array}{ll}
T_{0}^{(1)}(s)=d_{0}^{(1)}, & T_{j}^{(1)}(s)=(0,0,-d), \\
T_{0}^{(2)}(s)=d_{0}^{(2)}, & T_{j}^{(2)}(s)=(0,0,0),
\end{array}
$$


where all of the components are constants, and $T_{j}^{(1)}(s)$ and $T_{j}^{(2)}(s)$ represent the locations of each monopole. We have taken the 3 -space coordinate such that the monopoles are located along the 3 -direction. The boundary Nahm data $W$ is given by a quaternion, compatible with matching conditions (2.4a), (2.4b). Here we can choose $W=\lambda \mathbf{1}_{2}, \lambda \in \mathbb{R}$, and the unit vector $\hat{\boldsymbol{\omega}}$ is chosen to be $(0,0, \pm 1)$ without loss of generality and find the matching condition is

$$
\frac{1}{2} \lambda^{2}(\hat{\boldsymbol{\omega}})_{3}=d \Rightarrow \pm \frac{1}{2} \lambda^{2}=d
$$

As can be seen from the HS calorons, the boundary Nahm data are corresponding to the scales of monopoles. Thus, we find that the matching conditions link the scale parameter and the distance parameter of the constituent monopole cores, for the calorons with nontrivial holonomy, in general. In another word, the large scale limit (LSL) simultaneously induces the large distance limit (LDL).

The gauge potential $A_{\mu}$ in the configuration space $\mathbb{R}^{3} \times S^{1}$ can be obtained from the Nahm transform, see appendix B for this construction in detail. We firstly consider the LSL of the $(1,1)$-calorons. Due to the matching condition (2.14), the LSL induces the LDL, i.e., $\lambda \rightarrow \infty \Leftrightarrow d \rightarrow \infty$, so that the bulk Nahm data $T_{j}^{(1)}$ diverges. This means that the Nahm transform is not well-defined in this limit. In fact, the explicit calculation shows that the gauge potential of $(1,1)$-calorons in the LDL turns out to be,

$$
A_{0}=\frac{1}{2}\left(\mu_{0}-3 \mu\right) \sigma_{3}, A_{j}=0
$$

which, of course, is a pure gauge, thus does not have magnetic charge. We find from (2.15) that the holonomy parameter still survives in this limit, and the holonomy (2.2) is maximal when $\mu=\mu_{0} / 6$. We refer to the limit as "purely holonomic" gauge field. As we will see in the following, the order of the large scale limit and the massless limit is significant matter, in general.

In order to obtain the calorons with net magnetic charge from KvBLL, we have to decouple the large scale limits and the large distance limits. Thus, we take the holonomy parameter $\mu \rightarrow 0$ in advance, i.e., to make the interval $I_{1}$ be shrinking to zero, which leads to $(1,[1])$-calorons. We can also take the limit $\mu \rightarrow \mu_{0} / 2$, which shrinks $I_{2} \rightarrow 0$, and find both are equivalent due to the rotation map. These are obviously the Harrington-Shepard 1-caloron $\left(\mathrm{HS}_{1}\right)$, considered in the last subsection. In these cases, the matching conditions become trivial, namely no special relation exists between $\lambda$ and $d$, thus the LSL does not induce the LDL. From the analysis in the previous subsection, the magnetic charge appears only for the $\mathrm{JNR}_{1}$. In summarize, we find the following diagram for various limits from $(1,1)$-calorons:

\section{$2.3 \quad(2,2)$-calorons}

Next, we consider $(2,2)$-calorons and their various large scale limits and the appearance of magnetic charges. The general form of the bulk Nahm data is given in terms of Jacobi 


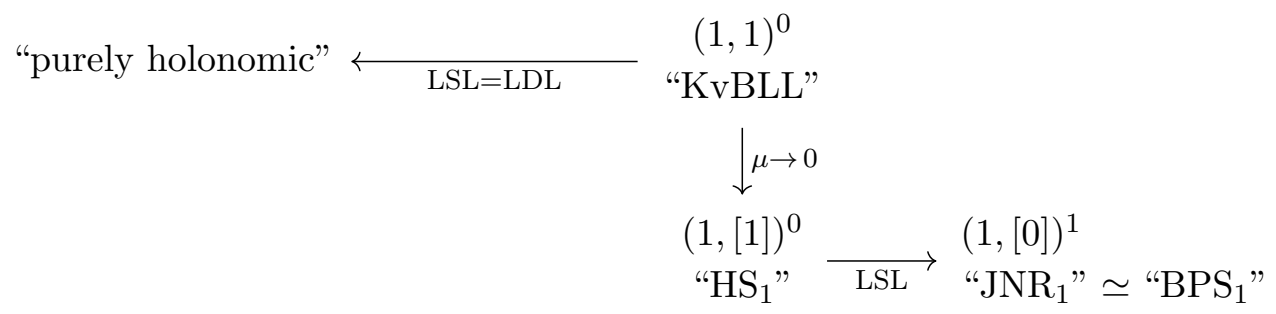

Figure 1. Several limits from the KvBLL calorons, where we denote the magnetic charge $Q$ at the superscript $(*, *)^{Q}$.

elliptic function $[19,20]^{1}$

$$
\begin{aligned}
& T_{1}^{(m)}(s)=e^{i h^{(m)}(s) \sigma_{2}}\left(f_{1}^{(m)}(s) \sigma_{1}+g_{1}^{(m)}(s) \sigma_{3}\right)+d_{1}^{(m)} \mathbf{1}_{2}=: \mathcal{F}_{1}^{(m)} \sigma_{1}+\mathcal{G}_{1}^{(m)} \sigma_{3}+d_{1}^{(m)} \mathbf{1}_{2}, \\
& T_{2}^{(m)}(s)=f_{2}^{(m)}(s) \sigma_{2}+d_{2}^{(m)} \mathbf{1}_{2}, \\
& T_{3}^{(m)}(s)=e^{i h^{(m)}(s) \sigma_{2}}\left(g_{3}^{(m)}(s) \sigma_{1}+f_{3}^{(m)}(s) \sigma_{3}\right)+d_{3}^{(m)} \mathbf{1}_{2}=: \mathcal{G}_{3}^{(m)} \sigma_{1}+\mathcal{F}_{3}^{(m)} \sigma_{3}+d_{3}^{(m)} \mathbf{1}_{2}, \\
& T_{0}^{(m)}(s)=\frac{1}{2} \frac{d h^{(m)}}{d s}(s) \sigma_{2}+d_{0}^{(m)} \mathbf{1}_{2},
\end{aligned}
$$

where

$$
\begin{aligned}
& \mathcal{F}_{1}^{(m)}=f_{1}^{(m)}(s) \cos h^{(m)}(s)-g_{1}^{(m)}(s) \sin h^{(m)}(s), \\
& \mathcal{G}_{1}^{(m)}=g_{1}^{(m)}(s) \cos h^{(m)}(s)+f_{1}^{(m)}(s) \sin h^{(m)}(s), \\
& \mathcal{G}_{3}^{(m)}=g_{3}^{(m)}(s) \cos h^{(m)}(s)-f_{3}^{(m)}(s) \sin h^{(m)}(s), \\
& \mathcal{F}_{3}^{(m)}=f_{3}^{(m)}(s) \cos h^{(m)}(s)+g_{3}^{(m)}(s) \sin h^{(m)}(s),
\end{aligned}
$$

and

$$
\begin{aligned}
f_{1}^{(m)}(s) & =\frac{D_{m} k_{m}^{\prime}}{\operatorname{cn} 2 D_{m}\left(s-s_{m}\right)} \cos \varphi_{m}, \quad g_{1}^{(m)}(s)=-D_{m} \frac{\operatorname{dn} 2 D_{m}\left(s-s_{m}\right)}{\operatorname{cn} 2 D_{m}\left(s-s_{m}\right)} \sin \varphi_{m}, \\
f_{2}^{(m)}(s) & =-D_{m} k_{m}^{\prime} \frac{\operatorname{sn} 2 D_{m}\left(s-s_{m}\right)}{\operatorname{cn} 2 D_{m}\left(s-s_{m}\right)} \\
g_{3}^{(m)}(s) & =\frac{D_{m} k_{m}^{\prime}}{\operatorname{cn} 2 D_{m}\left(s-s_{m}\right)} \sin \varphi_{m}, \quad f_{3}^{(m)}(s)=D_{m} \frac{\operatorname{dn} 2 D_{m}\left(s-s_{m}\right)}{\operatorname{cn} 2 D_{m}\left(s-s_{m}\right)} \cos \varphi_{m},
\end{aligned}
$$

with sn, cn, dn are Jacobi elliptic functions, $k_{m}(m=1,2)$ are their modulus parameters, $k_{m}^{\prime}:=\sqrt{1-k_{m}^{2}}$, and $s_{1}=0, s_{2}= \pm \mu_{0} / 2$. Although the differentiable even functions $h^{(m)}(s)$ are appeared in the most general solution to the system, we consider the cases $h^{(m)}(s)=0$ here.

${ }^{1}$ The solutions in [19] are not completely the most general form of the bulk data of monopole charge 2 . In fact, the $h^{(m)}(s)$ dependence was ignored there. 
The boundary data is quaternion valued 2-vector $W=(p, q) \in \mathbb{H}^{2}$, which are constrained by the matching conditions $(2.4 \mathrm{a}),(2.4 \mathrm{~b})$. They are written down by the quaternion components of $p$ and $q$, i.e., $\left(p_{0}, \boldsymbol{p}\right),\left(q_{0}, \boldsymbol{q}\right) \in \mathbb{R}^{4}$ as

$$
\begin{aligned}
\frac{1}{2} \operatorname{Tr} \sigma_{j} W^{\dagger} P_{ \pm} W & = \pm \mathcal{S}_{j} \sigma_{1}+\mathcal{A}_{j} \sigma_{2} \pm \mathcal{U}_{j} \sigma_{3} \pm \Delta_{j} \mathbf{1}_{2} \\
& = \pm \mathcal{S}_{j} \sigma_{1}+\mathcal{A}_{j} \sigma_{2} \pm \mathcal{M}_{\uparrow, j}(p) \sigma_{\uparrow} \pm \mathcal{M}_{\downarrow, j}(q) \sigma_{\downarrow}
\end{aligned}
$$

where

$$
\begin{aligned}
\mathcal{S}_{j} & =\frac{1}{2}\left\{p_{0} q_{0} \hat{\omega}_{j}+p_{0}(\hat{\boldsymbol{\omega}} \times \boldsymbol{q})_{j}+q_{0}(\hat{\boldsymbol{\omega}} \times \boldsymbol{p})_{j}+(\hat{\boldsymbol{\omega}} \cdot \boldsymbol{p}) q_{j}-(\boldsymbol{p} \cdot \boldsymbol{q}) \hat{\omega}_{j}+(\hat{\boldsymbol{\omega}} \cdot \boldsymbol{q}) p_{j}\right\}, \\
\mathcal{A}_{j} & =\frac{1}{2}\left\{p_{0} q_{j}-q_{0} p_{j}-(\boldsymbol{p} \times \boldsymbol{q})_{j}\right\}, \\
\mathcal{M}_{\uparrow, j}(p) & =\frac{1}{2}\left(p_{0}^{2}-\boldsymbol{p}^{2}\right) \hat{\omega}_{j}+p_{0}(\hat{\boldsymbol{\omega}} \times \boldsymbol{p})_{j}+(\hat{\boldsymbol{\omega}} \cdot \boldsymbol{p}) p_{j}, \\
\mathcal{M}_{\downarrow, j}(q) & =\frac{1}{2}\left(q_{0}^{2}-\boldsymbol{q}^{2}\right) \hat{\omega}_{j}+q_{0}(\hat{\boldsymbol{\omega}} \times \boldsymbol{q})_{j}+(\hat{\boldsymbol{\omega}} \cdot \boldsymbol{q}) q_{j},
\end{aligned}
$$

with $\mathcal{U}_{j}=\frac{1}{2}\left(\mathcal{M}_{\uparrow, j}(p)-\mathcal{M}_{\downarrow, j}(q)\right)$ and $\Delta_{j}=\frac{1}{2}\left(\mathcal{M}_{\uparrow, j}(p)+\mathcal{M}_{\downarrow, j}(q)\right)$, and we have defined

$$
\sigma_{\uparrow}:=\left(\begin{array}{ll}
1 & 0 \\
0 & 0
\end{array}\right), \sigma_{\downarrow}:=\left(\begin{array}{ll}
0 & 0 \\
0 & 1
\end{array}\right) .
$$

As in the case of $(1,1)$-calorons, the large scale limits, i.e., $|p|,|q| \rightarrow \infty$, and the large distance limits $\left|d_{j}^{(m)}\right| \rightarrow \infty$ are linked each other due to these matching conditions. Thus, those limits lead to the divergence of the constant parts of the bulk data $d_{j}^{(m)}$, so that the bulk Nahm data are not well-defined. We expect that those limits of the $(2,2)$-calorons turn out to be pure gauge or purely holonomic similarly to the KvBLL calorons, which should be confirmed by the numerical Nahm transform in the future.

In order to find the magnetic limits, we firstly take the limit to the trivial holonomy, i.e., the massless case, as previously. In such cases, we find from (2.5) that the $\hat{\boldsymbol{\omega}}$ dependence in the matching conditions are switched off, and the decoupling of the large scale limit (LSL) and the large distance limit (LDL) occurs. We choose the monopole on $I_{2}$, say, be massless, and find the Nahm data of $\left([2], 2_{k_{1}}\right)$-calorons, where the modulus $k_{1}$ dependence is shown explicitly for later convenience, and there is no $k_{2}$ dependence obviously. The Nahm transform to this ([2], $\left.2_{k_{1}}\right)$-calorons is performed numerically in [31].

We are now able to take LSL from the massless $\left([2], 2_{k_{1}}\right)$-calorons defined on the interval $I_{1}=\left(-\mu_{0} / 2, \mu_{0} / 2\right)$ provided that the elliptic functions diverge at $s \rightarrow \pm \mu_{0} / 2$, due to the matching conditions (2.5). In order to fulfill this condition, the period of the elliptic functions is "coincident" with the range of $I_{1}$ exactly. In order to make this coincidence, the modulus $k_{1}$ and the parameter $D_{1}$ have to take special values, and we call it a resonance, "Res" in short. In fact, we can find the bulk data in the resonance have simple poles and the residues be an irreducible representation of $s u(2)$ at $s= \pm \mu_{0} / 2$. We, therefore, observe that the Nahm data satisfy the monopole conditions, which give the BPS 2-monopole $\left(\mathrm{BPS}_{2}\right)$ in this case [32]. 


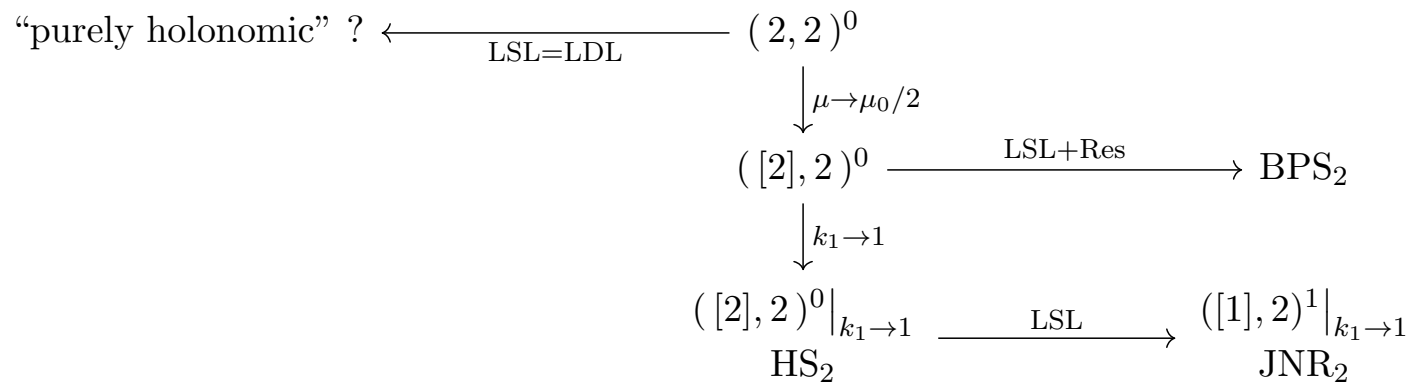

Figure 2. Several magnetically charged limits from the $(2,2)$-caloron.

In addition to the $\mathrm{BPS}_{2}$ monopole limit, we can find another magnetically charged limit through the Harrington-Shepard 2-caloron $\left(\mathrm{HS}_{2}\right)$. Namely, if we further take the modulus $k_{1} \rightarrow 1$ limit of the neutral ([2], $2_{k_{1}}$ )-calorons, we obtain $\mathrm{HS}_{2}$ calorons, still being magnetically neutral. The $k_{1} \rightarrow 1$ limit of the bulk Nahm data turns out to be

$$
T_{1}^{(1)}=-D_{1} \sin \varphi_{1} \sigma_{3}, T_{2}^{(1)}=0, T_{3}^{(1)}=D_{1} \cos \varphi_{1} \sigma_{3},
$$

where we choose the spatial origin such that $d_{j}^{(1)}=0, j=1,2,3$. By taking $\varphi_{1}=0$, or making a spatial rotation, we get the bulk data

$$
T_{1}^{(1)}=T_{2}^{(1)}=0, T_{3}^{(1)}=D_{1} \sigma_{3} .
$$

This is the bulk Nahm data of $\mathrm{HS}_{2}$. We remark that only the diagonal part of the bulk data is alive, namely this can be written as a direct sum form. We use this fact in the following sections. The matching conditions are given by (2.5), and the right hand sides are thus trivially zero. We find that it is sufficient to take $W=(p, q) \in \mathbb{H}^{2}$ with $q$ is proportional to $p$, i.e., $q=\alpha p, \alpha \in \mathbb{R}$. For the detail of the Nahm transform of $\mathrm{HS}_{2}$, see appendix A.

As we have already seen that the magnetic $\mathrm{JNR}_{2}$ caloron is the large scale limit of the $\mathrm{HS}_{2}$ caloron, we have found another path of the charged limit from $(2,2)$-calorons.

The sequence of the magnetic limits from $(2,2)$-calorons is depicted as the following diagram. In this diagram, the left and center columns are neutral and the right is magnetically charged cases. We remark that the $\mathrm{BPS}_{2}$ has magnetic charge 2 , whereas the $\mathrm{JNR}_{2}$ has 1 , respectively.

\section{Intrinsically charged calorons}

So far, we have observed that the large scale limits of magnetically neutral calorons often possess net magnetic charges. The remarkable fact is that all of the charged cases considered are induced from massless ones, i.e., having no holonomy parameter. In contrast to these mechanism, it is known that there exist calorons which are endowed with intrinsic magnetic charges. The ADHM/Nahm construction for such calorons is given by considering monopoles of unbalanced charges $N$ and $N^{\prime}$ with $N>N^{\prime}$ in each interval $I_{2}$ and 
$I_{1}$, respectively. Then we find that the composite objects, $\left(N, N^{\prime}\right)$-calorons, have magnetic charge $Q=N-N^{\prime}$ naturally. By construction, these $\left(N, N^{\prime}\right)$-calorons are still massive, i.e., the holonomy parameter is alive.

In this section, we consider the $(2,1)$-calorons as a simple illustration for such intrinsically charged calorons with non-trivial holonomy. We expect that the $(2,1)$-calorons considered here have some connection with $[33,34]$.

\section{$3.1(2,1)$-calorons}

\subsubsection{ADHM/Nahm construction for $\left(N, N^{\prime}\right)$-calorons}

First of all, we summarize the ADHM/Nahm construction for the intrinsically charged calorons, in which the constituent monopoles have different charges $N$ and $N^{\prime}$ with $N>$ $N^{\prime}$. Namely, the bulk Nahm data on $I_{m}(m=2,1)$ are $u(N)$ - and $u\left(N^{\prime}\right)$-valued matrices $T_{\nu}^{(m)}(s),(\nu=1,2,3,0)$, respectively. They independently satisfy the Nahm equations (2.3) on each interval. The boundary Nahm data are given by a rectangular $N \times N^{\prime}$ matrix $X$ enjoying $X^{\dagger} X=1_{N^{\prime}}$. The matching conditions are

$$
\begin{aligned}
X^{\dagger} T_{j}^{(2)}(\mu) X & =T_{j}^{(1)}(\mu), \\
{ }^{t} X T_{j}^{(2)}\left(\mu_{0}-\mu\right) \bar{X} & =T_{j}^{(1)}(-\mu),
\end{aligned}
$$

in place of $(2.4 \mathrm{a}),(2.4 \mathrm{~b})$, where $\bar{X}$ stands for the complex conjugate matrix.

We remark that there are gauge transformations for the $\left(N, N^{\prime}\right)$-Nahm data, $g_{2}(s) \in$ $\mathrm{U}(N), s \in I_{2}$ and $g_{1}(s) \in \mathrm{U}\left(N^{\prime}\right), s \in I_{1}$, with the reality conditions $g_{2}(-s)=\bar{g}_{2}(s)$ and $g_{1}(-s)=\bar{g}_{1}(s)$ are understood. They act for the Nahm data as

$$
\begin{aligned}
T_{j}^{(2)}(s) & \mapsto g_{2}(s) T_{j}^{(2)}(s) g_{2}^{-1}(s), T_{0}^{(2)}(s) \mapsto g_{2}(s) T_{0}^{(2)}(s) g_{2}^{-1}(s)+i g_{2}(s) \frac{d}{d s} g_{2}^{-1}(s), \\
T_{j}^{(1)}(s) & \mapsto g_{1}(s) T_{j}^{(1)}(s) g_{1}^{-1}(s), T_{0}^{(1)}(s) \mapsto g_{1}(s) T_{0}^{(1)}(s) g_{1}^{-1}(s)+i g_{1}(s) \frac{d}{d s} g_{1}^{-1}(s), \\
X & \mapsto g_{2}(\mu) X g_{1}^{-1}(\mu) .
\end{aligned}
$$

\subsubsection{The Nahm data for general $(2,1)$-calorons}

We now make a specification $\left(N, N^{\prime}\right)=(2,1)$ and give its Nahm data definitely. The Nahm data include that of $(2,1)$-calorons with $\mathrm{U}(1)$-symmetry obtained in [18] as a special case. The Nahm transform of these $\mathrm{U}(1)$-symmatric cases is considered in the next subsection.

The bulk data $T_{\nu}^{(2)}(s)$ on the interval $I_{2}$ are given in terms of the general 2-monopole data already appeared in the (2,2)-calorons (2.16a), (2.16b), (2.16c), (2.16d), while $T_{\nu}^{(1)}(s):=t_{\nu}^{(1)}$ on $I_{1}$ are

$$
\begin{aligned}
t_{\nu}^{(1)}(s) & =\left(d_{1}^{(1)}, d_{2}^{(1)}, d_{3}^{(1)}, d_{0}^{(1)}\right) \\
& :=\left(d_{\downarrow, 1}, d_{\downarrow, 2}, d_{\downarrow, 3}, d_{\downarrow, 0}\right) \in \mathbb{R}^{4},
\end{aligned}
$$

where $d_{\downarrow, \nu}$ 's are constants due to the Nahm equations (2.3), and the notation is introduced for later convenience. 
The boundary data is $2 \times 1$ complex matrix $X$ with two real parameters, such as $X={ }^{t}\left(\cos \beta e^{i \alpha}, \sin \beta\right)$ where $\alpha \in[0,2 \pi)$ and $\beta \in[0, \pi]$, without loss of generality. Thus, we find the matching conditions

$$
\begin{aligned}
X^{\dagger} T_{j}^{(2)}(\mu) X & =t_{j}^{(1)}(\mu), \\
{ }^{t} X T_{j}^{(2)}\left(\mu_{0}-\mu\right) \bar{X} & =t_{j}^{(1)}(-\mu),
\end{aligned}
$$

are given by

$$
\begin{aligned}
\mathcal{F}_{1}^{(2)}(\mu) \sin 2 \beta \cos \alpha+\mathcal{G}_{1}^{(2)}(\mu) \cos 2 \beta & =d_{\downarrow, 1}-d_{1}^{(2)}=: \delta_{1}, \\
-f_{2}^{(2)}(\mu) \sin 2 \beta \sin \alpha & =d_{\downarrow, 2}-d_{2}^{(2)}=: \delta_{2}, \\
\mathcal{G}_{3}^{(2)}(\mu) \sin 2 \beta \cos \alpha+\mathcal{F}_{3}^{(2)}(\mu) \cos 2 \beta & =d_{\downarrow, 3}-d_{3}^{(2)}=: \delta_{3},
\end{aligned}
$$

where we have used the fact

$$
\begin{aligned}
& X^{\dagger} \sigma_{1} X={ }^{t} X \sigma_{1} \bar{X}=\sin 2 \beta \cos \alpha, \\
& X^{\dagger} \sigma_{2} X=-{ }^{t} X \sigma_{2} \bar{X}=-\sin 2 \beta \sin \alpha, \\
& X^{\dagger} \sigma_{3} X={ }^{t} X \sigma_{3} \bar{X}=\cos 2 \beta .
\end{aligned}
$$

Note that the matching conditions (3.4a) and (3.4b) are equivalent provided that $\mathcal{F}_{1}^{(2)}, \mathcal{G}_{1}^{(2)}, \mathcal{F}_{3}^{(2)}$ and $\mathcal{G}_{3}^{(2)}$ are even functions and $f_{2}^{(2)}(s)$ is odd function with respect to $s_{2}=\mu_{0} / 2$. The requirements are fulfilled due to $(2.18 \mathrm{a}),(2.18 \mathrm{~b}),(2.18 \mathrm{c})$, and $h^{(2)}$ is even.

Eliminating $\alpha$ and $\beta$ from (3.5a), (3.5b), (3.5c), we find the matching condition is

$$
\frac{\left(\mathcal{F}_{3}^{(2)}(\mu) \delta_{1}-\mathcal{G}_{1}^{(2)}(\mu) \delta_{3}\right)^{2}+\left(-\mathcal{G}_{3}^{(2)}(\mu) \delta_{1}+\mathcal{F}_{1}^{(2)}(\mu) \delta_{3}\right)^{2}}{\left(\mathcal{F}_{1}^{(2)}(\mu) \mathcal{F}_{3}^{(2)}(\mu)-\mathcal{G}_{1}^{(2)}(\mu) \mathcal{G}_{3}^{(2)}(\mu)\right)^{2}}+\left(\frac{\delta_{2}}{f_{2}^{(2)}(\mu)}\right)^{2}=1
$$

which can be simplified if we take $h^{(2)}(s)=\varphi_{2}=0$,

$$
\left(\frac{\delta_{1}}{f_{1}^{(2)}(\mu)}\right)^{2}+\left(\frac{\delta_{2}}{f_{2}^{(2)}(\mu)}\right)^{2}+\left(\frac{\delta_{3}}{f_{3}^{(2)}(\mu)}\right)^{2}=1
$$

\subsection{Nahm transform for (2,1)-calorons with U(1)-symmetry}

We now restrict the $(2,1)$-calorons with $\mathrm{U}(1)$ or $\mathrm{SO}(2)$-symmetric cases [18]. The $G$ symmetric calorons for some symmetric group $G \subseteq \mathrm{SO}(3)$ are constructed from the $G$ symmetric Nahm data $[18,20,30]$ with invariance under an action of $G$. For the Nahm data of $(2,1)$-calorons, the $G$-symmetry are defined as the covariance under the map, for the bulk data on $I_{2}$

$$
\begin{aligned}
& T_{j}^{(2)} \mapsto R_{2} T_{j}^{(2)} R_{2}^{-1}=R_{j k} T_{k}^{(2)}, \\
& T_{0}^{(2)} \mapsto R_{2} T_{0}^{(2)} R_{2}^{-1}=T_{0}^{(2)},
\end{aligned}
$$


on $I_{1}$

$$
\begin{aligned}
t_{j}^{(1)} & \mapsto R_{j k} t_{k}^{(1)}, \\
t_{0}^{(1)} & \mapsto t_{0}^{(1)},
\end{aligned}
$$

and the boundary data is transformed trivially $X \mapsto X$, where $R_{2}$ is a two-dimensional irreducible representation of $G$, and $R_{j k}$ is $\mathrm{SO}(3)$-matrices. The fact that the simultaneous transformation $\sigma_{j} \mapsto R_{2^{\prime}} \sigma_{j} R_{2^{\prime}}^{-1}=R_{j k} \sigma_{k}$ with another irreducible representation of $R_{2^{\prime}} \in$ $\mathrm{SU}(2)$ leads to the invariance of $T_{j}^{(2)} \otimes \sigma_{j}$ and $t_{j}^{(1)} \otimes \sigma_{j}$, we find the corresponding calorons are $G$-invariant. Here we consider the case that $G$ is a $\mathrm{U}(1)$ subgroup of $\mathrm{SU}(2)$, i.e., a rotation around the 3 -axis.

The U(1)-symmetric Nahm data can be obviously given by taking the $k_{2} \rightarrow 1$ limit of the bulk data on $I_{2}$ as in the case of $\mathrm{HS}_{2}$ limit from $(2,2)$-calorons. In this limit, only $f_{3}^{(2)}(s)$ remains non-zero, and we find from $(2.17 \mathrm{a})-(2.17 \mathrm{~d})$

$$
\begin{aligned}
& \mathcal{F}_{1}^{(2)}(s)=\mathcal{G}_{1}^{(2)}(s)=f_{2}^{(2)}(s)=0, \\
& \mathcal{G}_{3}^{(2)}(s)=-D_{2} \sin h^{(2)}, \quad \mathcal{F}_{3}^{(2)}(s)=D_{2} \cos h^{(2)} .
\end{aligned}
$$

Defining $D:=D_{2}$ and $h:=h^{(2)}$, we obtain the bulk data on $I_{2}$

$$
T_{1}^{(2)}=d_{1}^{(2)} \mathbf{1}_{2}, T_{2}^{(2)}=d_{2}^{(2)} \mathbf{1}_{2}, T_{3}^{(2)}=-D \sin h \sigma_{1}+D \cos h \sigma_{3}+d_{3}^{(2)} \mathbf{1}_{2}
$$

together with $T_{0}^{(2)}=d_{0}^{(2)} 1_{2}$, and no modification is necessary for the $I_{1}$ bulk data. In order to perform the Nahm transform, we gauge (3.12) into

$$
T_{1}^{(2)}=d_{1}^{(2)} \mathbf{1}_{2}, T_{2}^{(2)}=d_{2}^{(2)} \mathbf{1}_{2}, T_{3}^{(2)}=D \sigma_{3}+d_{3}^{(2)} \mathbf{1}_{2},
$$

by an appropriate $g_{2} \in \mathrm{U}(2)$. Thus, the matching conditions (3.5a), (3.5b), (3.5c) are

$$
d_{\downarrow, 1}-d_{1}^{(2)}=d_{\downarrow, 2}-d_{2}^{(2)}=0, D \cos 2 \beta=d_{\downarrow, 3}-d_{3}^{(2)}=\delta_{3},
$$

which is independent of $\alpha$, so that we can fix $\alpha=0$.

The components of the bulk Nahm data of $(2,1)$-calorons with U(1)-symmetry (3.14) are constants as in the previously considered cases. Thus, it is expected that the Nahm transform can be performed analytically, and we show that it is possible, in this subsection.

First of all, we choose the coordinate origin such that the center of 2-monopole is located at the origin and all of the monopoles are aligned on the 3 -axis, and take a gauge $T_{0}^{(m)}=0$. Namely, we consider the Nahm data

$$
\begin{aligned}
T_{\nu}^{(2)} & =\left(0,0, D \sigma_{3}, 0\right) \\
t_{\nu}^{(1)} & =\left(0,0, d_{\downarrow, 3}, 0\right)=:\left(0,0, d_{\downarrow}, 0\right),
\end{aligned}
$$

where $\nu=(1,2,3,0)$ and we define $d_{\downarrow}:=d_{\downarrow, 3}$ for simplicity.

The bulk Weyl equations for each interval $I_{2}, I_{1}$ are

$$
\begin{aligned}
\left(i \frac{d}{d s}+\left(T_{\nu}^{(2)}+x_{\nu} \mathbf{1}_{2}\right) \otimes e_{\nu}\right) \boldsymbol{v}^{(2)}(s) & =0, s \in I_{2} \\
\left(i \frac{d}{d s}+\left(t_{\nu}^{(1)}+x_{\nu}\right) \otimes e_{\nu}\right) v^{(1)}(s) & =0, s \in I_{1},
\end{aligned}
$$


where $e_{\nu}=\left(-i \sigma_{1},-i \sigma_{2},-i \sigma_{3}, 1_{2}\right)$ is quaternion basis. These spinors are necessary to be normalized as,

$$
\int_{I_{2}} \boldsymbol{v}^{(2)}(s)^{\dagger} \boldsymbol{v}^{(2)}(s) d s+\int_{I_{1}} v^{(1)}(s)^{\dagger} v^{(1)}(s) d s=1_{2}
$$

For this purpose, we define the spinors in each interval as products of "interval-wise normalized" spinors and some quaternions $C_{1}, C_{2}$ and $B$, such that

$$
\begin{aligned}
& \boldsymbol{v}^{(2)}(s)=\left[\begin{array}{l}
\tilde{v}_{1}^{(2)}(s) C_{1} \\
\tilde{v}_{2}^{(2)}(s) C_{2}
\end{array}\right], \\
& v^{(1)}(s)=\tilde{v}^{(1)}(s) B .
\end{aligned}
$$

Note that there is no "boundary" part $U$ in contrast to the neutrally charged cases.

The components of the interval-wise normalized spinors are determined by the normalization conditions

$$
\int_{I_{2}} \tilde{v}_{1}^{(2)}(s)^{\dagger} \tilde{v}_{1}^{(2)}(s) d s=1_{2}, \int_{I_{2}} \tilde{v}_{2}^{(2)}(s)^{\dagger} \tilde{v}_{2}^{(2)}(s) d s=1_{2}, \int_{I_{1}} \tilde{v}^{(1)}(s)^{\dagger} \tilde{v}^{(1)}(s) d s=1_{2} .
$$

Thus, we find

$$
\begin{aligned}
& \tilde{v}_{1}^{(2)}(s)=\frac{1}{\sqrt{N_{+}}} e^{i x_{0}\left(s-\mu_{0} / 2\right)}\left(1_{2} \cosh r_{+}\left(s-\mu_{0} / 2\right)-\left(\hat{\boldsymbol{x}}_{+} \cdot \boldsymbol{\sigma}\right) \sinh r_{+}\left(s-\mu_{0} / 2\right)\right), \\
& \tilde{v}_{2}^{(2)}(s)=\frac{1}{\sqrt{N_{-}}} e^{i x_{0}\left(s-\mu_{0} / 2\right)}\left(1_{2} \cosh r_{-}\left(s-\mu_{0} / 2\right)-\left(\hat{\boldsymbol{x}}_{-} \cdot \boldsymbol{\sigma}\right) \sinh r_{-}\left(s-\mu_{0} / 2\right)\right), \\
& \tilde{v}^{(1)}(s)=\frac{1}{\sqrt{N_{\downarrow}}} e^{i x_{0} s}\left(1_{2} \cosh r_{\downarrow} s-\left(\hat{\boldsymbol{x}}_{\downarrow} \cdot \boldsymbol{\sigma}\right) \sinh r_{\downarrow} s\right),
\end{aligned}
$$

where $\boldsymbol{x}_{ \pm}=(x, y, z \mp D), \boldsymbol{x}_{\downarrow}=\left(x, y, z-d_{\downarrow}\right), N_{ \pm}=\frac{\sinh 2 r_{ \pm}\left(\mu_{0} / 2-\mu\right)}{r_{ \pm}}$and $N_{\downarrow}=\frac{\sinh 2 r_{\downarrow} \mu}{r_{\downarrow}}$, with $r_{ \pm}:=\left|\boldsymbol{x}_{ \pm}\right|$and $r_{\downarrow}:=\left|\boldsymbol{x}_{\downarrow}\right|$.

The gauge connection is formally written as

$$
\begin{aligned}
A_{\mu}= & i B^{\dagger} \partial_{\mu} B+i B^{\dagger} \int_{I_{1}} \tilde{v}^{(1)}(s)^{\dagger} \partial_{\mu} \tilde{v}^{(1)}(s) d s B \\
& +i C_{1}^{\dagger} \partial_{\mu} C_{1}+i C_{1}^{\dagger} \int_{I_{2}} \tilde{v}_{1}^{(2)}(s)^{\dagger} \partial_{\mu} \tilde{v}_{1}^{(2)}(s) d s C_{1} \\
& +i C_{2}^{\dagger} \partial_{\mu} C_{2}+i C_{2}^{\dagger} \int_{I_{2}} \tilde{v}_{2}^{(2)}(s)^{\dagger} \partial_{\mu} \tilde{v}_{2}^{(2)}(s) d s C_{2},
\end{aligned}
$$

so it is necessary to determine $C_{1}, C_{2}$ and $B$ explicitly.

The matching conditions for the Weyl spinors are given in terms of the matching matrix $X$ as follows

$$
\begin{aligned}
X^{\dagger} \otimes 1_{2} \boldsymbol{v}^{(2)}(\mu) & =v^{(1)}(\mu), \\
{ }^{t} X \otimes 1_{2} \boldsymbol{v}^{(2)}\left(\mu_{0}-\mu\right) & =v^{(1)}(-\mu),
\end{aligned}
$$


which are equivalent to

$$
\begin{aligned}
\tilde{v}_{1}^{(2)}(\mu) C_{1} \cos \beta+\tilde{v}_{2}^{(2)}(\mu) C_{2} \sin \beta & =\tilde{v}^{(1)}(\mu) B, \\
\tilde{v}_{1}^{(2)}\left(\mu_{0}-\mu\right) C_{1} \cos \beta+\tilde{v}_{2}^{(2)}\left(\mu_{0}-\mu\right) C_{2} \sin \beta & =\tilde{v}^{(1)}(-\mu) B .
\end{aligned}
$$

From these matching conditions, we can find $C_{1}$ and $C_{2}$ in terms of $B$ as,

$$
\begin{aligned}
& C_{1} \cos \beta=q_{-} B, \\
& C_{2} \sin \beta=q_{+} B,
\end{aligned}
$$

where

$$
\begin{aligned}
& q_{-}=\sqrt{\frac{N_{+}}{N_{\downarrow}}} \frac{1}{M}\left(-\boldsymbol{m} \cdot \boldsymbol{a}_{-} 1_{2}+i\left(a_{-, 0} \boldsymbol{m}-\boldsymbol{m} \times \boldsymbol{a}_{-}\right) \cdot \boldsymbol{\sigma}\right), \\
& q_{+}=\sqrt{\frac{N_{-}}{N_{\downarrow}}} \frac{1}{M}\left(\boldsymbol{m} \cdot \boldsymbol{a}_{+} 1_{2}-i\left(a_{+, 0} \boldsymbol{m}-\boldsymbol{m} \times \boldsymbol{a}_{+}\right) \cdot \boldsymbol{\sigma}\right),
\end{aligned}
$$

with

$$
\begin{aligned}
\boldsymbol{m}= & \cosh r_{-}\left(\frac{\mu_{0}}{2}-\mu\right) \sinh r_{+}\left(\frac{\mu_{0}}{2}-\mu\right) \hat{\boldsymbol{x}}_{+}-\sinh r_{-}\left(\frac{\mu_{0}}{2}-\mu\right) \cosh r_{+}\left(\frac{\mu_{0}}{2}-\mu\right) \hat{\boldsymbol{x}}_{-}, \\
M:= & \boldsymbol{m}^{2} \\
= & \frac{1}{2}\left(\cosh 2 r_{-}\left(\frac{\mu_{0}}{2}-\mu\right) \cosh 2 r_{+}\left(\frac{\mu_{0}}{2}-\mu\right)-1\right. \\
& \left.\quad-\sinh 2 r_{-}\left(\frac{\mu_{0}}{2}-\mu\right) \sinh 2 r_{+}\left(\frac{\mu_{0}}{2}-\mu\right) \hat{\boldsymbol{x}}_{+} \cdot \hat{\boldsymbol{x}}_{-}\right), \\
a_{ \pm, 0}= & \sin \frac{\mu_{0} x_{0}}{2}\left(\cosh r_{ \pm}\left(\frac{\mu_{0}}{2}-\mu\right) \cosh r_{\downarrow} \mu+\hat{\boldsymbol{x}}_{ \pm} \cdot \hat{\boldsymbol{x}}_{\downarrow} \sinh r_{ \pm}\left(\frac{\mu_{0}}{2}-\mu\right) \sinh r_{\downarrow} \mu\right), \\
\boldsymbol{a}_{ \pm}= & \cos \frac{\mu_{0} x_{0}}{2}\left(\hat{\boldsymbol{x}}_{ \pm} \sinh r_{ \pm}\left(\frac{\mu_{0}}{2}-\mu\right) \cosh r_{\downarrow} \mu+\hat{\boldsymbol{x}}_{\downarrow} \cosh r_{ \pm}\left(\frac{\mu_{0}}{2}-\mu\right) \sinh r_{\downarrow} \mu\right) \\
& +\sin \frac{\mu_{0} x_{0}}{2} \sinh r_{ \pm}\left(\frac{\mu_{0}}{2}-\mu\right) \sinh r_{\downarrow} \mu \hat{\boldsymbol{x}}_{ \pm} \times \hat{\boldsymbol{x}}_{\downarrow} .
\end{aligned}
$$

It is necessary to make the normalization condition (3.17) accomplish. The condition is equvalent to

$$
B^{\dagger} B+C_{1}^{\dagger} C_{1}+C_{2}^{\dagger} C_{2}=1_{2} .
$$

Let us take a gauge choice such that $B$ is proportional to $1_{2}$, and define $B=\frac{1}{\sqrt{N}} 1_{2}$, then we obtain

$$
N=1+\frac{q_{-}^{\dagger} q_{-}}{\cos ^{2} \beta}+\frac{q_{+}^{\dagger} q_{+}}{\sin ^{2} \beta} .
$$

Here we use $N$ for the normalization function, and do not confuse with the monopole charges. From (3.25a), (3.25b) and the following definitions, we have

$$
\begin{aligned}
q_{ \pm}^{\dagger} q_{ \pm}=\frac{N_{\mp}}{2 N_{\downarrow} M}( & \cosh 2 r_{ \pm}\left(\frac{\mu_{0}}{2}-\mu\right) \cosh 2 r_{\downarrow} \mu-\cos \mu_{0} x_{0} \\
& \left.+\sinh 2 r_{ \pm}\left(\frac{\mu_{0}}{2}-\mu\right) \sinh 2 r_{\downarrow} \mu \hat{\boldsymbol{x}}_{ \pm} \cdot \hat{\boldsymbol{x}}_{\downarrow}\right) .
\end{aligned}
$$


In this gauge choice, $C_{1}$ and $C_{2}$ are simply given by

$$
\begin{aligned}
& C_{1} \cos \beta=\frac{1}{\sqrt{N}} q_{-}, \\
& C_{2} \sin \beta=\frac{1}{\sqrt{N}} q_{+} .
\end{aligned}
$$

Substituting these formulas into (3.21), we finally obtain

$$
\begin{aligned}
A_{\mu}= & i \sqrt{N}\left(\partial_{\mu} \frac{1}{\sqrt{N}}\right) \mathbf{1}_{2}+\frac{i}{N} \int_{I_{1}} \tilde{v}^{(1)}(s)^{\dagger} \partial_{\mu} \tilde{v}^{(1)}(s) d s \\
& +\frac{i}{N \cos ^{2} \beta} q_{-}^{\dagger} \partial_{\mu} q_{-}+\frac{i}{N \cos ^{2} \beta} q_{-}^{\dagger} \int_{I_{2}} \tilde{v}_{1}^{(2)}(s)^{\dagger} \partial_{\mu} \tilde{v}_{1}^{(2)}(s) d s q_{-} \\
& +\frac{i}{N \sin ^{2} \beta} q_{+}^{\dagger} \partial_{\mu} q_{+}+\frac{i}{N \sin ^{2} \beta} q_{+}^{\dagger} \int_{I_{2}} \tilde{v}_{2}^{(2)}(s)^{\dagger} \partial_{\mu} \tilde{v}_{2}^{(2)}(s) d s q_{+} .
\end{aligned}
$$

This is the gauge connection of $(2,1)$-calorons with $\mathrm{U}(1)$-symmetry in analytic form. We have confirmed that the gauge configuration (3.31) satisfies the ASD conditions, and $A_{\mu} \in$ $s u(2)$, i.e., the trace term is cancelled, with Mathematica, as expected. We can make a plot of the action density, $\operatorname{Tr} F_{\nu \rho} F_{\nu \rho}$, of $(2,1)$-calorons from (3.31), for several values of the moduli parameter $\beta$ in figures 3 and 4 .

At the limiting case $\beta \rightarrow \pi / 2$ (or $0, \pi$ ), only the terms including $q_{-}$(or $q_{+}$) survive so that the analysis becomes quite simple. In this case, it is convenient to choose a gauge $B=\tilde{q}_{-}^{\dagger} / \sqrt{N}=: q_{-} /\left|q_{-}\right| \sqrt{N}$ where $\tilde{q}_{-}$is a unit quaternion, i.e., $\tilde{q}_{-}^{\dagger} \tilde{q}_{-}=1_{2}$. In this limit, we find $N \cos ^{2} \beta \rightarrow\left|q_{-}\right|^{2}$, then $C_{1} \rightarrow 1_{2}$. Hence the gauge connection turns out to be

$$
A_{\mu}=i \int_{I_{2}} \tilde{v}_{1}^{(2)}(s)^{\dagger} \partial_{\mu} \tilde{v}_{1}^{(2)}(s) d s
$$

whose components are given as

$$
\begin{aligned}
& A_{0}=\left(-\frac{1}{2 r_{-}}+\left(\frac{\mu_{0}}{2}-\mu\right) \operatorname{coth} 2 r_{-}\left(\frac{\mu_{0}}{2}-\mu\right)\right)\left(\hat{\boldsymbol{x}}_{-} \cdot \boldsymbol{\sigma}\right) \underset{r \rightarrow \infty}{\longrightarrow}\left(\frac{\mu_{0}}{2}-\mu-\frac{1}{2 r}\right)(\hat{\boldsymbol{x}} \cdot \boldsymbol{\sigma}), \\
& A_{j}=\left(\frac{1}{2 r_{-}}-\frac{\frac{\mu_{0}}{2}-\mu}{\sinh 2 r_{-}\left(\frac{\mu_{0}}{2}-\mu\right)}\right)\left(\hat{\boldsymbol{x}}_{-} \times \boldsymbol{\sigma}\right)_{j} .
\end{aligned}
$$

We find from the asymptotic behavior of $A_{0}$ that the gauge configuration has magnetic charge one, as expected. In addition, although this limit becomes translationally invariant with $x_{0}$-direction, as in the cases of BPS monopoles, this configuration has a non-trivial holonomy at infinity

$$
\Omega(\infty):=\lim _{r \rightarrow \infty} \mathcal{P} \exp i \int_{S^{1}} A_{0}(x) d x_{0}=\exp \left\{i \pi\left(1-\frac{2 \mu}{\mu_{0}}\right)(\hat{\boldsymbol{x}} \cdot \boldsymbol{\sigma})\right\},
$$

which can not be gauged to trivial holonomy with a periodic gauge transformation, unless $\mu=0$ or $\mu_{0} / 2$. The case of maximal holonomy is obtained when $\mu=\mu_{0} / 4$. We also find that the $\mu \rightarrow 0$ case turns out to be the $\mathrm{BPS}_{1}$ monopole, exactly, and the $\mu \rightarrow \mu_{0} / 2$ case tends to pure gauge. 
(1) $\beta=\pi / 8$

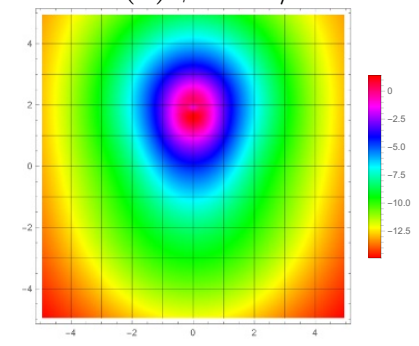

(5) $\beta=5 \pi / 8$

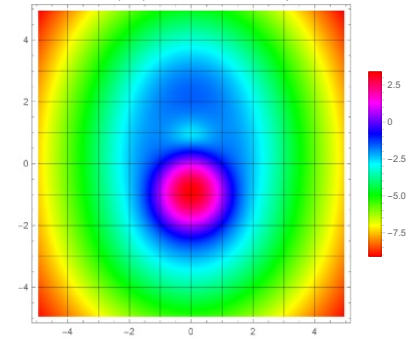

(2) $\beta=\pi / 4$

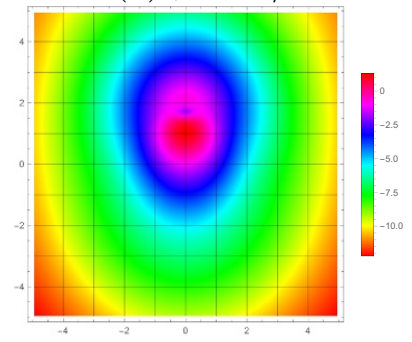

(4) $\beta=\pi / 2$

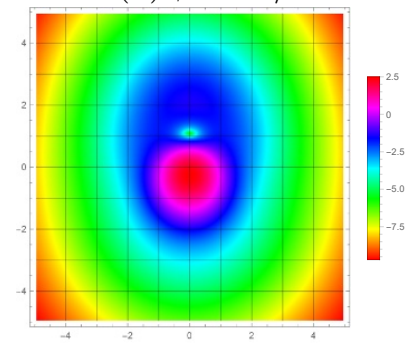

(6) $\beta=3 \pi / 4$

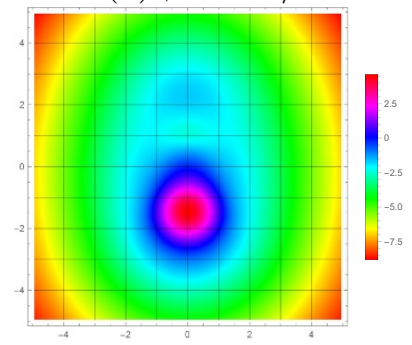

(3) $\beta=3 \pi / 8$

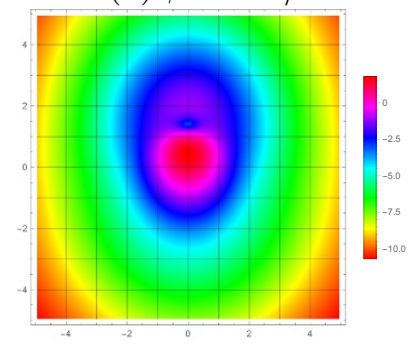

(7) $\beta=7 \pi / 8$

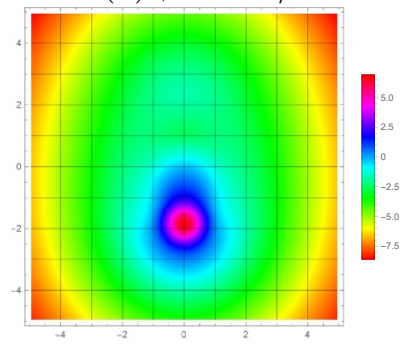

Figure 3. Action density plot in 1, 3-plane for some values of $\beta$ with $\log$-scale. The density is high (red), and low (orange).

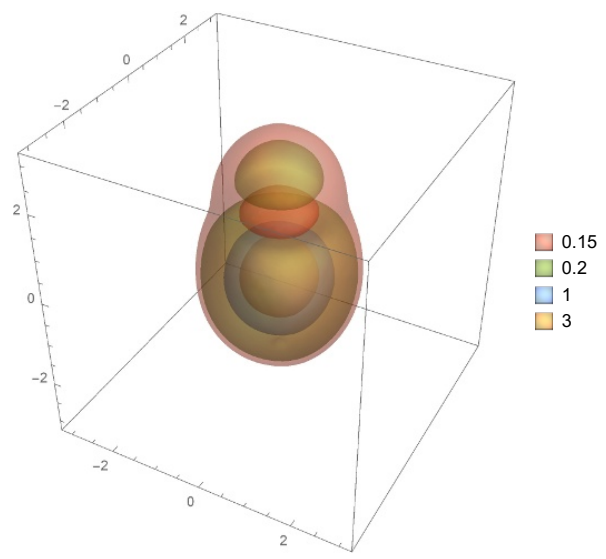

Figure 4. 3-dim. surfaces of constant action density for $\beta=\pi / 2$. 
Moreover, if we further take the limit of (3.33a), (3.33b) with $D \rightarrow \infty$, i.e., at the "large distance limit", then we find

$$
\lim _{D \rightarrow \infty} A_{0}=\left(\frac{\mu_{0}}{2}-\mu\right) \sigma_{3}, \lim _{D \rightarrow \infty} A_{j}=0
$$

due to the fact $r_{-} \sim D \rightarrow \infty$ and $\hat{\boldsymbol{x}}_{-} \rightarrow(0,0,1)$. This is also the purely holonomic configuration as in the large distance limit of the KvBLL calorons. Although it is very complicated, we can actually certify that the gauge configuration is purely holonomic as (3.35) at the limit $D \rightarrow \infty$ without fixing $\beta$.

We expect that there will be many circumstances with these purely holonomic configurations in calorons with non-trivial holonomy at the large distance limits.

\section{Decomposable Nahm data and removing limits}

In the last section, we have considered calorons with intrinsic magnetic charges, the $(2,1)$ calorons as a particular example. As we have already seen in section 2 that the magnetic charges appear as several kind of large scale limits from a magnetically neutral caloron. At this point, we can make a guess that the intrinsically charged calorons are also induced from some neutral one. In this section, we consider this structure in detail. First, we construct Nahm data of decomposable type, i.e., a superposition of some fundamental "monopoles", and secondly remove one of them to the spatial infinity referred to it as the removing limit. This monopole removing process have also been considered in slightly different context in [35] for $\mathrm{SU}(\mathcal{N})$-monopoles, in which a charge $N$-monopole data is approximated by a superposition of $N$ unit monopoles. Here we consider more exact situation, i.e., the cases of no approximation in the Nahm data.

\subsection{Nahm data of $(2,1 \oplus 1)$-calorons}

The concrete example we consider is $(2,1 \oplus 1)$-caloron and its removing limit. As defined in Introduction, $(1 \oplus 1)$ - "monopoles" are the exact superposition of two 1-monopoles, whose bulk Nahm data are give by $2 \times 2$ diagonal matrix, in another words a direct sum of two $1 \times 1$ matrices. In general, the $\left(m \oplus m^{\prime}\right)$ bulk data are constructed by the block diagonal matrices with a direct sum of $m \times m$ and $m^{\prime} \times m^{\prime}$ matrices. We remark that the bulk data of $\mathrm{HS}_{N}$ calorons are special cases of massless and decomposable data.

The most general form of the $(1 \oplus 1)$ bulk Nahm data is

$$
T_{\nu}^{(1)}(s)=\left(\begin{array}{cc}
d_{\uparrow, \nu} & 0 \\
0 & d_{\downarrow, \nu}
\end{array}\right)=\frac{1}{2}\left(d_{\uparrow, \nu}-d_{\downarrow, \nu}\right) \sigma_{3}+\frac{1}{2}\left(d_{\uparrow, \nu}+d_{\downarrow, \nu}\right) \mathbf{1}_{2},
$$

where all of the components $d_{\uparrow, \nu}$ and $d_{\downarrow, \nu}$ are constants. This bulk data obviously enjoys the Nahm equations and represents the superposition of two fundamental 1-monopoles located at $d_{\uparrow, \nu}$ and $d_{\downarrow, \nu}$, respectively. Adopting (4.1) as the bulk Nahm data on one of the interval, say, $I_{1}$, we can assemble the bulk Nahm data of $(2,1 \oplus 1)$-calorons by taking the 2-monopole (2.16a)-(2.16d) as the bulk data on $I_{2}$ together with appropriate boundary 
data of the form (2.19). The matching conditions at $s=\mu,(2.4 \mathrm{a})$, can be arranged into the matrix form such as

$$
\begin{aligned}
& {\left[\begin{array}{cccc}
\mathcal{F}_{1}^{(2)}(\mu) & 0 & \mathcal{G}_{1}^{(2)}(\mu) & d_{1}^{(2)} \\
0 & f_{2}^{(2)}(\mu) & 0 & d_{2}^{(2)} \\
\mathcal{G}_{3}^{(2)}(\mu) & 0 & \mathcal{F}_{3}^{(2)}(\mu) & d_{3}^{(2)}
\end{array}\right]-\left[\begin{array}{cccc}
0 & 0 & \frac{1}{2}\left(d_{\uparrow, 1}-d_{\downarrow, 1}\right) & \frac{1}{2}\left(d_{\uparrow, 1}+d_{\downarrow, 1}\right) \\
0 & 0 & \frac{1}{2}\left(d_{\uparrow, 2}-d_{\downarrow, 2}\right) & \frac{1}{2}\left(d_{\uparrow, 2}+d_{\downarrow, 2}\right) \\
0 & 0 & \frac{1}{2}\left(d_{\uparrow, 3}-d_{\downarrow, 3}\right) & \frac{1}{2}\left(d_{\uparrow, 3}+d_{\downarrow, 3}\right)
\end{array}\right]} \\
& =\left[\begin{array}{cccc}
\mathcal{S}_{1} & \mathcal{A}_{1} & \mathcal{U}_{1} & \Delta_{1} \\
\mathcal{S}_{2} & \mathcal{A}_{2} & \mathcal{U}_{2} & \Delta_{2} \\
\mathcal{S}_{3} & \mathcal{A}_{3} & \mathcal{U}_{3} & \Delta_{3}
\end{array}\right]
\end{aligned}
$$

or equivalently,

$$
\begin{aligned}
& {\left[\begin{array}{cccc}
\mathcal{F}_{1}^{(2)}(\mu) & 0 & \mathcal{G}_{1}^{(2)}(\mu)+d_{1}^{(2)} & -\mathcal{G}_{1}^{(2)}(\mu)+d_{1}^{(2)} \\
0 & f_{2}^{(2)}(\mu) & d_{2}^{(2)} & d_{2}^{(2)} \\
\mathcal{G}_{3}^{(2)}(\mu) & 0 & \mathcal{F}_{3}^{(2)}(\mu)+d_{3}^{(2)} & -\mathcal{F}_{3}^{(2)}(\mu)+d_{3}^{(2)}
\end{array}\right]-\left[\begin{array}{cccc}
0 & 0 & d_{\uparrow, 1} & d_{\downarrow, 1} \\
0 & 0 & d_{\uparrow, 2} & d_{\downarrow, 2} \\
0 & 0 & d_{\uparrow, 3} & d_{\downarrow, 3}
\end{array}\right] } \\
= & {\left[\begin{array}{cccc}
\mathcal{S}_{1} & \mathcal{A}_{1} & \mathcal{M}_{\uparrow, 1} & \mathcal{M}_{\downarrow, 1} \\
\mathcal{S}_{2} & \mathcal{A}_{2} & \mathcal{M}_{\uparrow, 2} & \mathcal{M}_{\downarrow, 2} \\
\mathcal{S}_{3} & \mathcal{A}_{3} & \mathcal{M}_{\uparrow, 3} & \mathcal{M}_{\downarrow, 3}
\end{array}\right], }
\end{aligned}
$$

where the rows are corresponding to each space component $j=1,2,3$ of the Nahm matrices, and the columns are each component of the matrix basis $\left\{\sigma_{1}, \sigma_{2}, \sigma_{3}, \mathbf{1}_{2}\right\}$ in (4.2), and $\left\{\sigma_{1}, \sigma_{2}, \sigma_{\uparrow}, \sigma_{\downarrow}\right\}$ in (4.3), respectively. The remaining matching conditions at $s=-\mu=$ $\mu_{0}-\mu,(2.4 \mathrm{~b})$, are automatically satisfied due to the reality conditions of the bulk data.

The final task is to find the boundary data satisfying the matching conditons (4.2) and (4.3), i.e., the entries of the right hand sides. What we need now is to demonstrate the existence of the well-defined limit from neutral $(2,1 \oplus 1)$-calorons to a magnetic $(2,1)$ caloron. Thus, it is not necessary to determine the general form to the boundary data for the present purpose.

We now find an explicit illustration of $(2,1)$-caloron as a removing limit. From the matching conditions (4.2) and (4.3), it has to be $\mathcal{S}_{2}=\mathcal{A}_{1}=\mathcal{A}_{3}=0$. In addition, we impose the conditions $\mathcal{U}_{2}=\Delta_{2}=0$, or equivalently $\mathcal{M}_{\uparrow, 2}=\mathcal{M}_{\downarrow, 2}=0$, in order to find such example. These type of the matching conditions have already been considered in [19] for neutral $(2,2)$-calorons, referred to as the parallel and coplanar type (type- $\mathrm{P}_{\mathrm{cop}}$ ). Taking the identical parametrization for the boundary data with [19], we find

$$
\begin{aligned}
\mathcal{S}_{1} & =\frac{1}{2} \lambda \rho \sin (\eta \mp \psi), & \mathcal{S}_{3} & =\frac{1}{2} \lambda \rho \cos (\eta \mp \psi), \\
\mathcal{A}_{2} & = \pm \frac{1}{2} \lambda \rho \sin \psi, & & \\
\mathcal{M}_{\uparrow, 1} & =\frac{1}{2} \lambda^{2} \sin \eta, & \mathcal{M}_{\downarrow, 1} & =\frac{1}{2} \rho^{2} \sin (\eta \mp 2 \psi), \\
\mathcal{M}_{\uparrow, 3} & =\frac{1}{2} \lambda^{2} \cos \eta, & \mathcal{M}_{\downarrow, 3} & =\frac{1}{2} \rho^{2} \cos (\eta \mp 2 \psi),
\end{aligned}
$$

where the parameters $\lambda, \rho, \eta$ and $\psi$ are defined as follows. We can take the coponents of $W$ in the left hand side of (2.19) as $W=\left(\lambda \mathbf{1}_{2}, \rho \hat{q}\right)$, where $\lambda, \rho>0$ and $\hat{q}$ a unit quaternion. 
This restriction is always possible due to a gauge degrees of freedom for $W$ under $W \rightarrow \hat{h} W$, where $\hat{h}$ is an arbitrary unit quaternion. For the type- $\mathrm{P}_{\text {cop }}$ boundary data, it is appropriate to take a parametrisation for the unit quaternion $\hat{q}$ in its components,

$$
\left(\hat{q}_{1}, \hat{q}_{2}, \hat{q}_{3}, \hat{q}_{0}\right)=(0, \sin \psi, 0, \cos \psi)
$$

and for the unit vector $\hat{\boldsymbol{\omega}}$ in the projection operator $P_{ \pm}$

$$
\hat{\omega}=(\sin \eta, 0, \cos \eta)
$$

Substituting these into the boundary data (2.20)-(2.23), we find $\mathcal{S}_{2}=\mathcal{A}_{1}=\mathcal{A}_{3}=\mathcal{M}_{\uparrow, 2}=$ $\mathcal{M}_{\downarrow, 2}=0$, together with (4.4a)-(4.4d).

We should confirm the matching conditions have a solution with appropriate values of the parameters. Focusing on (4.3), it is necessary that $d_{2}^{(2)}=d_{\uparrow, 2}=d_{\downarrow, 2}$, and the remaining conditions are

$$
\begin{aligned}
\mathcal{F}_{1}^{(2)}(\mu) & =\frac{1}{2} \lambda \rho \sin (\eta \mp \psi), \quad \mathcal{G}_{3}^{(2)}(\mu)=\frac{1}{2} \lambda \rho \cos (\eta \mp \psi), \\
f_{2}^{(2)}(\mu) & = \pm \frac{1}{2} \lambda \rho \sin \psi
\end{aligned}
$$

and

$$
\begin{aligned}
\mathcal{G}_{1}^{(2)}(\mu)+d_{1}^{(2)}-d_{\uparrow, 1} & =\frac{1}{2} \lambda^{2} \sin \eta, \\
-\mathcal{G}_{1}^{(2)}(\mu)+d_{1}^{(2)}-d_{\downarrow, 1} & =\frac{1}{2} \rho^{2} \sin (\eta \mp 2 \psi), \\
\mathcal{F}_{3}^{(2)}(\mu)+d_{3}^{(2)}-d_{\uparrow, 3} & =\frac{1}{2} \lambda^{2} \cos \eta, \\
-\mathcal{F}_{3}^{(2)}(\mu)+d_{3}^{(2)}-d_{\downarrow, 3} & =\frac{1}{2} \rho^{2} \cos (\eta \mp 2 \psi) .
\end{aligned}
$$

From the conditions (4.7a) and (4.7b), we can eliminate the parameters $\lambda, \rho$ and $\eta$, and find

$$
f_{2}^{(2)}(\mu)^{2}=\left(\mathcal{F}_{1}^{(2)}(\mu)^{2}+\mathcal{G}_{3}^{(2)}(\mu)^{2}\right) \sin ^{2} \psi
$$

Substituting the 2-monopole bulk data (2.17a)-(2.17d) and (2.18a)-(2.18c) into (4.9), we observe that it is necessary

$$
k_{2}^{\prime 2} \operatorname{sn}^{2} 2 D_{2}\left(\mu-\mu_{0} / 2\right)=\left(k_{2}^{\prime 2} \cos ^{2} h^{(2)}(\mu)+\operatorname{dn}^{2} 2 D_{2}\left(\mu-\mu_{0} / 2\right) \sin ^{2} h^{(2)}(\mu)\right) \sin ^{2} \psi,
$$

for arbitrary $\varphi_{2}$. It is obvious that there exists a parameter $\psi$ in accordance with the constraint (4.10) due to the fact $k_{2}^{\prime 2} \leq k_{2}^{\prime 2} \cos ^{2} h^{(2)}(\mu)+\operatorname{dn}^{2} 2 D_{2}\left(\mu-\mu_{0} / 2\right) \sin ^{2} h^{(2)}(\mu) \leq 1$ and $0 \leq k_{2}^{\prime 2} \operatorname{sn}^{2} 2 D_{2}\left(\mu-\mu_{0} / 2\right) \leq k_{2}^{\prime 2}$. Thus, we have found that the conditions (4.7a) and (4.7b) are consistent for arbitrary $\lambda, \rho$ and $\eta$ with given $\mu, k_{2}, D_{2}$ and $h^{(2)}(\mu)$. Finally, we choose the "location" parameters $d_{j}^{(2)}, d_{\uparrow, j}, d_{\downarrow, j},(j=1,3)$ such that the constraints $(4.8 \mathrm{a})-(4.8 \mathrm{~d})$ hold, and find that the matching conditions for $(2,1 \oplus 1)$-calorons with type- $\mathrm{P}_{\text {cop }}$ boundary data have a consistent solution. 


\subsection{Removing limit to $(2,1)$-caloron Nahm data}

We next consider the removing limit to a magnetically charged $(2,1)$-caloron Nahm data, from the $(2,1 \oplus 1)$-calorons constructed in the last subsection.

The removing limit can be taken by letting one of the location of the "monopole" in the decomposable $(1 \oplus 1)$-monopole send to spatial infinity. The spatial "locations" of the decomposable monopole are $d_{\uparrow, j}$ and $d_{\downarrow, j},(j=1,2,3)$, respectively. We choose, for instance, some components of the upper part $d_{\uparrow, j}$ to be large. From the matching conditions (4.8a) and (4.8c), the removing limit $\left|d_{\uparrow, 1}\right|,\left|d_{\uparrow, 3}\right| \rightarrow \infty$ inevitably requires $\lambda \rightarrow \infty$, since the other boundary values of the bulk data in (4.8a) and (4.8c) have to be finite. We also find the remaining component $\left|d_{\uparrow, 2}\right|$ must keep finite value from the condition $d_{2}^{(2)}=d_{\uparrow, 2}=d_{\downarrow, 2}$. Thus, we observe that the removing limit is derived when the upper part monopole is thrown far away on the $(1,3)$-plane from the core region. It is necessary to show the consistency between the removing limit and the other matching conditions (4.7a), (4.7b), (4.8b) and (4.8d). We firstly find, from (4.7a) and (4.7b), that the product $\lambda \rho$ has to be finite when $\lambda \rightarrow \infty$, because the left hand sides are finite. Hence, we have to take the scaling limit, i.e., $\lambda \rightarrow \infty$ with $\rho \rightarrow 0$ and $\lambda \rho \rightarrow C \in \mathbb{R}$, for the consistent matching conditions of the removing limit. Consequently, the matching conditions for the removing limit $\left|d_{\uparrow, 1}\right|,\left|d_{\uparrow, 3}\right| \rightarrow \infty$ are

$$
\begin{aligned}
\mathcal{F}_{1}^{(2)}(\mu) & =\frac{1}{2} C \sin (\eta \mp \psi), \quad \mathcal{G}_{3}^{(2)}(\mu)=\frac{1}{2} C \cos (\eta \mp \psi), \\
f_{2}^{(2)}(\mu) & = \pm \frac{1}{2} C \sin \psi, \\
-\mathcal{G}_{1}^{(2)}(\mu)+d_{1}^{(2)}-d_{\downarrow, 1} & =0 \\
-\mathcal{F}_{3}^{(2)}(\mu)+d_{3}^{(2)}-d_{\downarrow, 3} & =0 .
\end{aligned}
$$

The first two conditions are equivalent to (4.7a) and (4.7b) replacing $\lambda \rho$ with $C$, so that we have already shown the existence of the solutions. Comparing the last two conditions with (3.5a) and (3.5c), we observe that they are equivalent to the case $\beta=\pi / 2$, which is consistent with $(3.5 \mathrm{~b})$ due to $d_{2}^{(2)}=d_{\downarrow, 2}$. We have consequently found that the $(2,1 \oplus 1)$ caloron has a one-monopole removing limit into the $(2,1)$-caloron with particular moduli parameters. Since there still remain moduli parameters in the $I_{2}$ bulk data, the field configuration in this removing limit has a structure similar to those of figure 3 .

Although the decomposable Nahm data considered above is not a general $(2,1 \oplus 1)$ caloron, this aspect is common for all the removing limits. This comes from the fact that the matching conditions for $(2,1)$-calorons are providing combined relations of independent matrix components in $T_{j}^{(2)}$, while those for $(2,1 \oplus 1)$-calorons are not. At this point, we remark that a restricted case of $(2,2)$-caloron in large scale has $(2,1)$-caloron limit [18]. We expect that this case corresponds to a special case of the removing limit considered in this seciton, in which a partial large scale limit can be taken. In addition, we can easily find that there exist $(1 \oplus 1)$-Nahm data (4.1) which can not be derived from a 2-monopole Nahm data. It is also possible to find consistent matching conditions for the assembled $(2,1 \oplus 1)$ calorons and the removing limit from them. For these cases, the matching conditions 


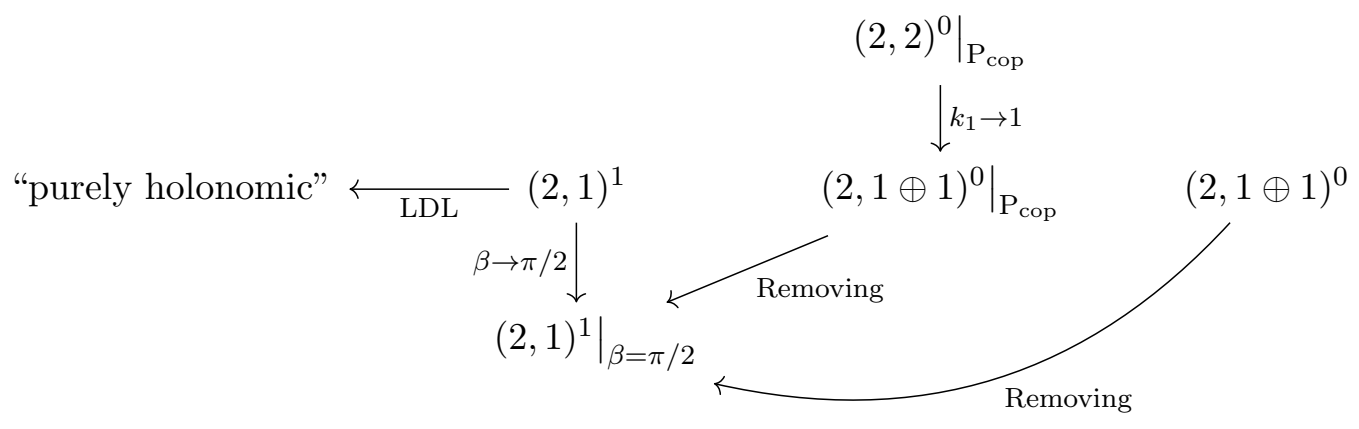

Figure 5. The removing limit from $(2,1 \oplus 1)$-calorons into $(2,1)$-calorons.

$\mathcal{M}_{\uparrow, 2}=\mathcal{M}_{\downarrow, 2}=0$ are no longer necessary and we can not apply the matching conditions of type- $\mathrm{P}_{\text {cop }}$. Similar consideration shows that the one-monopole removing limit also gives the $(2,1)$-calorons of $\beta=\pi / 2$ due to the reason mentioned above.

The schematic diagram of the removing limit is given in figure 5 . The most right $(2,1 \oplus 1)$-calorons represents the ones that the $(1 \oplus 1)$ part can not be derived from a 2-monopole.

Hence, we observe that not all of the intrinsically charged calorons can be obtained from the neutral and decomposable types, in general.

\section{Summary and outlook}

In summarize, we have considered the magnetically charged limits of calorons with and without holonomy parameters. It is found that all of the cases with magnetic charges are accompanied with large scale limits. From some specific examples, we have found that the magnetic charge appears through the following distinct approaches. First, by taking the large scale limit from a massless, or trivial holonomy caloron, we can obtain the BPS monopoles, which are translationally invariant with $S^{1}$ direction. In this process, the bulk Nahm data has to take "resonance", i.e., having single poles at the boundaries, simultaneously with the large scale limit. This can be seen in the case of $(2,2)$ calorons into $\mathrm{BPS}_{2}$. When the bulk Nahm data are constants just as in the case of $\mathrm{HS}_{N}$, we can also take the large scale limit into the $\mathrm{JNR}_{N}$, but having no translationally invariance with $S^{1}$. Finally, it is found that the removing limits from a neutral and decomposable caloron turn out to be a caloron with intrinsically magnetic charges with a partially large scale limit. In this case, the holonomy parameter is still alive and it is possible to have a non-trivial Polyakov loop.

As can be seen from figures 1 and 2, the order of the large scale limits and the massless limits is strongly significant, since the link between the scale and the distance parameters breaks down when the massless limit is taken. In addition, we have observed that there appear the cases of purely holonomic configurations from the large scale limit together with the large distance limit. The existence of these configurations, or states, would be 
significant in the perspective of physics, since the non-trivial holonomy is one of the criterion for distinguishing the confinement/deconfinement phase.

As an explicit illustration for the intrinsically charged calorons, we have considered the $(2,1)$-calorons in detail, which have magnetic charge 1 . The Nahm transform is performed, however, only for the U(1)-symmetric configurations. More general configurations can be visualized through the numerical Nahm transform, which is under development by the present authors. At this point, although the moduli space dimensions for magnetically neutral calorons are proved to be identical with that of instantons in $\mathbb{R}^{4}$ [36], the moduli space dimensions for the intrinsically charged calorons are unknown. The determination to the moduli space dimensions of general $\left(N, N^{\prime}\right)$-calorons still remains for a future work.

In most cases, the magnetically charged calorons being considered so far possess magnetic charge 1, i.e., $\left(N, N^{\prime}\right)$-calorons of $N=N^{\prime}+1$ with and without holonomy parameters. Actually, there has been not known the Nahm data of calorons with $N>N^{\prime}+1$. Also, from the perspective of numerical Nahm transform under construction, there is some qualitative difference between the $N=N^{\prime}+1$ and $N>N^{\prime}+1$ cases. We should clarify that the distinction is crucial or not in the forthcoming paper.

\section{A Harrington-Shepard 2-caloron}

When $\mu \rightarrow \mu_{0} / 2$ (or 0 ), the $(2,2)$-calorons become massless ([2],2)-calorons. If we further take the modulus parameter $k$ of the bulk data in $I_{1}, k \rightarrow 1$, we obtain Harrington-Shepard type calorons of instanton number 2 .

The $k \rightarrow 1$ limit of the bulk data on $I_{1}$ and the boundary data $W$ are given as follows,

$$
\begin{aligned}
& T_{0}=T_{1}=T_{2}=0, \quad T_{3}=D \sin \phi \sigma_{1}+D \cos \phi \sigma_{3}, \\
& W=(p, q) \in \mathbb{H}^{2} .
\end{aligned}
$$

This can be gauge rotated with

$$
g=\left(\begin{array}{cc}
\cos \phi / 2 & -\sin \phi / 2 \\
\sin \phi / 2 & \cos \phi / 2
\end{array}\right), g^{\dagger}=g^{-1},
$$

into

$$
\begin{aligned}
T_{0}^{\prime} & =T_{1}^{\prime}=T_{2}^{\prime}=0, \quad T_{3}^{\prime}=g^{-1} T_{3} g=D \sigma_{3}, \\
W^{\prime} & =W g=(p \cos \phi / 2+q \sin \phi / 2,-p \sin \phi / 2+q \cos \phi / 2)=\left(p^{\prime}, q^{\prime}\right),
\end{aligned}
$$

where $T_{\nu}:=T_{\nu}^{(1)}$ and the boundary data is transformed in consistent with the matching conditions (2.5). Since the bulk data is given by constant matrices, the matching conditions (2.5) should be trivial. Thus, it is sufficient to take the boundary data, e.g., $p^{\prime}=\left(\boldsymbol{p}^{\prime}, 0\right)=\left(p_{1}, 0, p_{3}, 0\right)$ and $q^{\prime}=\left(\boldsymbol{q}^{\prime}, 0\right)=\left(q_{1}, 0, q_{3}, 0\right)$. This means $\boldsymbol{q}^{\prime}=\alpha \boldsymbol{p}^{\prime}$ for some $\alpha \in \mathbb{R}$. The matching conditions are then trivially satisfied: there is no relation between the bulk and the boundary Nahm data. 
From the Nahm data (A.4a), (A.4b), we perform the Nahm transform analytically to construct the components of the gauge potential. First of all, we consider the Weyl spinor $V$ with "boundary component" $U$ and "bulk spinor" $\boldsymbol{v}$

$$
V=\left[\begin{array}{c}
U \\
\boldsymbol{v}
\end{array}\right]=\left[\begin{array}{c}
U \\
\tilde{v}_{1}(s) C_{1} \\
\tilde{v}_{2}(s) C_{2}
\end{array}\right],
$$

where the components of bulk spinor are explicitly shown in the right hand side. The bulk Weyl equations are

$$
\left(i \frac{d}{d s}+T_{3}^{\prime} \otimes\left(-i \sigma_{3}\right)+\mathbf{1}_{2} \otimes x\right) \boldsymbol{v}=0 .
$$

The components of the solutions to (A.6) with partial normalization factors are

$$
\begin{aligned}
& \tilde{v}_{1}(s)=\frac{1}{\sqrt{N_{1}}} e^{i x_{0} s}\left(1_{2} \cosh r_{+} s-\left(\hat{\boldsymbol{x}}_{+} \cdot \boldsymbol{\sigma}\right) \sinh r_{+} s\right), \\
& \tilde{v}_{2}(s)=\frac{1}{\sqrt{N_{2}}} e^{i x_{0} s}\left(1_{2} \cosh r_{-} s-\left(\hat{\boldsymbol{x}}_{-} \cdot \boldsymbol{\sigma}\right) \sinh r_{-} s\right),
\end{aligned}
$$

where $r_{ \pm}=\sqrt{x^{2}+y^{2}+(z \mp D)^{2}}, N_{1}=\sinh \mu_{0} r_{+} / r_{+}$and $N_{2}=\sinh \mu_{0} r_{-} / r_{-}$. The matching conditions for these spinors are

$$
\begin{aligned}
& i W^{\dagger} U=\boldsymbol{v}\left(-\frac{\mu_{0}}{2}\right)-\boldsymbol{v}\left(\frac{\mu_{0}}{2}\right) \\
& \Leftrightarrow\left\{\begin{array}{l}
i p^{\dagger} U=\left\{\tilde{v}_{1}\left(-\frac{\mu_{0}}{2}\right)-\tilde{v}_{1}\left(\frac{\mu_{0}}{2}\right)\right\} C_{1} \\
i q^{\dagger} U=\left\{\tilde{v}_{2}\left(-\frac{\mu_{0}}{2}\right)-\tilde{v}_{2}\left(\frac{\mu_{0}}{2}\right)\right\} C_{2}
\end{array}\right. \\
& \Leftrightarrow\left\{\begin{array}{l}
C_{1}=i\left\{\tilde{v}_{1}\left(-\frac{\mu_{0}}{2}\right)-\tilde{v}_{1}\left(\frac{\mu_{0}}{2}\right)\right\}^{-1} p^{\dagger} U \\
C_{2}=i\left\{\tilde{v}_{2}\left(-\frac{\mu_{0}}{2}\right)-\tilde{v}_{2}\left(\frac{\mu_{0}}{2}\right)\right\}^{-1} q^{\dagger} U,
\end{array}\right.
\end{aligned}
$$

where we redefine $p^{\prime}=p$ and $q^{\prime}=q$. We introduce a normalization function $N$ and take a gauge

$$
U=\frac{p}{|p|} \frac{1}{\sqrt{N}} \Rightarrow p^{\dagger} U=\frac{|p|}{\sqrt{N}} 1_{2}, q^{\dagger} U=\frac{\alpha|p|}{\sqrt{N}} 1_{2}
$$

Thus we find

$$
\begin{aligned}
& C_{1}=-\frac{\sqrt{N_{1}}}{2 \sqrt{N}} \frac{2|p|}{\cosh \mu_{0} r_{+}-\cos \mu_{0} x_{0}}\left(1_{2} \sin \frac{\mu_{0} x_{0}}{2} \cosh \frac{\mu_{0} r_{+}}{2}-i \cos \frac{\mu_{0} x_{0}}{2} \sinh \frac{\mu_{0} r_{+}}{2}\left(\hat{\boldsymbol{x}}_{+} \cdot \boldsymbol{\sigma}\right)\right), \\
& C_{2}=-\frac{\alpha \sqrt{N_{2}}}{2 \sqrt{N}} \frac{2|p|}{\cosh \mu_{0} r_{-}-\cos \mu_{0} x_{0}}\left(1_{2} \sin \frac{\mu_{0} x_{0}}{2} \cosh \frac{\mu_{0} r_{-}}{2}-i \cos \frac{\mu_{0} x_{0}}{2} \sinh \frac{\mu_{0} r_{-}}{2}\left(\hat{\boldsymbol{x}}_{-} \cdot \boldsymbol{\sigma}\right)\right) .
\end{aligned}
$$

The overall normalization function $N$ is determined from

$$
(V, V)=U^{\dagger} U+\int_{I} \boldsymbol{v}^{\dagger} \boldsymbol{v} d s=1_{2}
$$


where the integration region is $I=\left(-\mu_{0} / 2, \mu_{0} / 2\right)$. This gives

$$
N=1+\frac{|p|^{2}}{2} \frac{\sinh \mu_{0} r_{+}}{r_{+}} \frac{1}{\cosh \mu_{0} r_{+}-\cos \mu_{0} x_{0}}+\frac{|q|^{2}}{2} \frac{\sinh \mu_{0} r_{-}}{r_{-}} \frac{1}{\cosh \mu_{0} r_{-}-\cos \mu_{0} x_{0}} .
$$

The gauge potential is obtained from this normalized Weyl spinor as

$$
\begin{aligned}
A_{\mu} & =i\left(V, \partial_{\mu} V\right) \\
& =i U^{\dagger} \partial_{\mu} U+i C_{1}^{\dagger} \int_{I} \tilde{v}_{1}^{\dagger} \partial_{\mu} \tilde{v}_{1} d s C_{1}+i N_{1} C_{1}^{\dagger} \partial_{\mu} C_{1}+i(1 \rightarrow 2) .
\end{aligned}
$$

A straightforward calculation shows

$$
\begin{aligned}
& A_{0}=-\frac{1}{4 N}\left\{|p|^{2} f_{+}\left(\hat{\boldsymbol{x}}_{+} \cdot \boldsymbol{\sigma}\right)+|q|^{2} f_{-}\left(\hat{\boldsymbol{x}}_{-} \cdot \boldsymbol{\sigma}\right)\right\}, \\
& A_{j}=\frac{1}{4 N}\left[|p|^{2}\left\{f_{+}\left(\hat{\boldsymbol{x}}_{+} \times \boldsymbol{\sigma}\right)_{j}+h_{+} \sigma_{j}\right\}+|q|^{2}\left\{f_{-}\left(\hat{\boldsymbol{x}}_{-} \times \boldsymbol{\sigma}\right)_{j}+h_{-} \sigma_{j}\right\}\right],
\end{aligned}
$$

where

$$
\begin{aligned}
f_{ \pm}= & \frac{\sinh \mu_{0} r_{ \pm}}{2 r_{ \pm}^{2}} \frac{2}{\cosh \mu_{0} r_{ \pm}-\cos \mu_{0} x_{0}} \\
& -\frac{\mu_{0}}{4 r_{ \pm}}\left(\frac{2}{\cosh \mu_{0} r_{ \pm}-\cos \mu_{0} x_{0}}\right)^{2}\left(1-\cosh \mu_{0} r_{ \pm} \cos \mu_{0} x_{0}\right), \\
h_{ \pm}= & \mu_{0} \frac{\sinh \mu_{0} r_{ \pm} \sin \mu_{0} x_{0}}{4 r_{ \pm}}\left(\frac{2}{\cosh \mu_{0} r_{ \pm}-\cos \mu_{0} x_{0}}\right)^{2} .
\end{aligned}
$$

This gauge potential yields the Harrington-Shepard type of instanton charge $2\left(\mathrm{HS}_{2}\right)$, i.e.,

$$
A_{\mu}=-\frac{1}{2} \eta_{\mu \nu} \partial_{\nu} \log N
$$

with $N$ given by (A.15). It is found that there appears no magnetic charge, i.e., $A_{0}(r) \sim$ $O\left(1 / r^{2}\right)$

The large scale limit of this gauge potential is obtained by taking $|p|^{2}=\alpha^{2}|q| \rightarrow \infty$, which leads

$$
\begin{aligned}
& A_{0}=-\frac{1}{4 N_{\infty}}\left\{f_{+}\left(\hat{\boldsymbol{x}}_{+} \cdot \boldsymbol{\sigma}\right)+\alpha^{2} f_{-}\left(\hat{\boldsymbol{x}}_{-} \cdot \boldsymbol{\sigma}\right)\right\}, \\
& A_{j}=\frac{1}{4 N_{\infty}}\left[\left\{f_{+}\left(\hat{\boldsymbol{x}}_{+} \times \boldsymbol{\sigma}\right)_{j}+h_{+} \sigma_{j}\right\}+\alpha^{2}\left\{f_{-}\left(\hat{\boldsymbol{x}}_{-} \times \boldsymbol{\sigma}\right)_{j}+h_{-} \sigma_{j}\right\}\right],
\end{aligned}
$$

where

$$
N_{\infty}=\frac{\sinh \mu_{0} r_{+}}{2 r_{+}} \frac{1}{\cosh \mu_{0} r_{+}-\cos \mu_{0} x_{0}}+\alpha^{2} \frac{\sinh \mu_{0} r_{-}}{2 r_{-}} \frac{1}{\cosh \mu_{0} r_{-}-\cos \mu_{0} x_{0}} .
$$

Note that the "separation parameter" $D$ is independent of the boundary data $p$ in this case, so that the large scale limit does not cause the large separation limit.

This is the JNR caloron of instanton charge $2\left(\mathrm{JNR}_{2}\right)$ appeared in the lower right part of figure 2. We find from the asymptotic behaviour of $A_{0}$ that the $\mathrm{JNR}_{2}$ has magnetic charge 1, i.e.,

$$
A_{0} \underset{r \rightarrow \infty}{\longrightarrow}-\frac{1}{2 r}(\hat{\boldsymbol{x}} \cdot \boldsymbol{\sigma})
$$




\section{B $\operatorname{KvBLL}(1,1)$-caloron}

The simplest calorons with nontrivial holonomy, i.e., $(1,1)$-calorons, are constructed independently by Kraan-van Baal [16] and Lee-Lu [17]. Here we give the procedure of the Nahm transform to reconstruct the gauge field from the Nahm data briefly, for convenience of further consideration.

The bulk and boundary Nahm data is given as

$$
\begin{aligned}
T_{j}^{(1)}(s) & =(0,0,-d), & T_{j}^{(2)}(s) & =(0,0,0), \\
W & =\lambda 1_{2}, & \hat{\boldsymbol{\omega}} & =(0,0, \pm 1) .
\end{aligned}
$$

Thus the matching conditions

$$
\begin{aligned}
& \frac{1}{2} \operatorname{Tr} \sigma_{j} W^{\dagger} P_{+} W=T_{j}^{(2)}(\mu)-T_{j}^{(1)}(\mu), \\
& \frac{1}{2} \operatorname{Tr} \sigma_{j} W^{\dagger} P_{-} W=T_{j}^{(1)}(-\mu)-T_{j}^{(2)}\left(\mu_{0}-\mu\right),
\end{aligned}
$$

turn out to be

$$
\pm \frac{1}{2} \lambda^{2}=d
$$

Hence we find that when the "separation" parameter $d$ diverges the scale parameter $\lambda$ does, simultaneously.

We now perform the Nahm transform to the $(1,1)$-Nahm data analytically. The Weyl spinor for $(1,1)$-calorons are given by

$$
V=\left[\begin{array}{c}
U \\
v_{1}(s), v_{2}(s)
\end{array}\right]=\left[\begin{array}{c}
U \\
\tilde{v}_{1}(s) C_{1}, \tilde{v}_{2}(s) C_{2}
\end{array}\right],
$$

where $v_{1}$ and $v_{2}$ are two-component vectors, and $C_{1}$ and $C_{2}$ are $2 \times 2$ matrices. Here, in the lower row, the spinor defined on $I_{1}$ is put on the left, and that on $I_{2}$ is on the right.

The bulk Weyl equations for spinors on each interval $I_{1}$ and $I_{2}$ are

$$
\begin{aligned}
& \left(i \frac{d}{d s}+y_{1}^{\dagger}\right) \tilde{v}_{1}(s)=0, \\
& \left(i \frac{d}{d s}+y_{2}^{\dagger}\right) \tilde{v}_{2}(s)=0,
\end{aligned}
$$

where $y_{1}=y_{1, \nu} e_{\nu}, y_{2}=y_{2, \nu} e_{\nu}$ with $y_{1, \nu}=y_{\nu}=\left(\boldsymbol{y}, y_{0}\right)=\left(x_{1}, x_{2}, x_{3}-d, x_{0}\right), y_{2, \nu}=x_{\nu}=$ $\left(\boldsymbol{x}, x_{0}\right)=\left(x_{1}, x_{2}, x_{3}, x_{0}\right)$. We make the "Weyl spinors" $\tilde{v}_{1}$ and $\tilde{v}_{2}$ be normalised on each interval, i.e.,

$$
\begin{aligned}
& \tilde{v}_{1}(s)=\frac{1}{\sqrt{N_{1}}} e^{i y_{1}^{\dagger} s}=\frac{1}{\sqrt{N_{1}}} e^{i y_{0} s} e^{-y_{j} \sigma_{j} s}, \\
& \tilde{v}_{2}(s)=\frac{1}{\sqrt{N_{2}}} e^{i y_{2}^{\dagger} \check{s}}=\frac{1}{\sqrt{N_{2}}} e^{i x_{0} \check{s}} e^{-x_{j} \sigma_{j} \check{s}},
\end{aligned}
$$


where $\check{s}=s-\mu_{0} / 2$ and

$$
\begin{array}{rlrl}
N_{1} & =\frac{\sinh 2 r_{1} \mu}{r_{1}}, & r_{1}:=\sqrt{y_{1}^{2}+y_{2}^{2}+y_{3}^{2}}=\sqrt{x_{1}^{2}+x_{2}^{2}+\left(x_{3}-d\right)^{2}}, \\
N_{2}=\frac{\sinh 2 r\left(\frac{\mu_{0}}{2}-\mu\right)}{r}, & r=\sqrt{x_{1}^{2}+x_{2}^{2}+x_{3}^{2}} .
\end{array}
$$

Next, we define spinors $v_{1}, v_{2}$ and $U$ satisfying the matching conditions

$$
\begin{aligned}
v_{2}(\mu)-v_{1}(\mu) & =(i \lambda) P_{+} U=: i \lambda U_{+}, \\
v_{1}(-\mu)-v_{2}\left(\mu_{0}-\mu\right) & =(i \lambda) P_{-} U=: i \lambda U_{-},
\end{aligned}
$$

where the new spinors are defined from the normalized spinors as $v_{1}(s)=\tilde{v}_{1}(s) C_{1}$ and $v_{2}(s)=\tilde{v}_{2}(s) C_{2}$, and $U$ is the upper part of the Weyl spinor. From these conditions, we find the matrices $C_{1}$ and $C_{2}$ are

$$
\begin{aligned}
& C_{1}=i \lambda A^{-1}\left(\tilde{v}_{2}^{-1}(\mu) U_{+}+\tilde{v}_{2}^{-1}\left(\mu_{0}-\mu\right) U_{-}\right), \\
& C_{2}=i \lambda B^{-1}\left(\tilde{v}_{1}^{-1}(\mu) U_{+}+\tilde{v}_{1}^{-1}(-\mu) U_{-}\right),
\end{aligned}
$$

where

$$
\begin{aligned}
A^{-1} & =\frac{i}{2} \sqrt{\frac{N_{1}}{N_{2}}} \tilde{A}^{-1}, \\
B^{-1} & =\frac{i}{2} \sqrt{\frac{N_{2}}{N_{1}}} \tilde{B}^{-1},
\end{aligned}
$$

with quaternions

$$
\begin{gathered}
\tilde{A}^{-1}=\frac{1_{2} a_{0} \sin \frac{\mu_{0}}{2} x_{0}-i\left(\operatorname{Re} e^{-i \frac{\mu_{0}}{2} x_{0}} \boldsymbol{a}\right) \cdot \boldsymbol{\sigma}}{a_{0}^{2} \sin ^{2} \frac{\mu_{0}}{2} x_{0}+\left|\operatorname{Re} e^{-i \frac{\mu_{0}}{2} x_{0}} \boldsymbol{a}\right|^{2}}, \\
\tilde{B}^{-1}=\frac{1_{2} a_{0} \sin \frac{\mu_{0}}{2} x_{0}-i\left(\operatorname{Re} e^{-i \frac{\mu_{0}}{2} x_{0}} \overline{\boldsymbol{a}}\right) \cdot \boldsymbol{\sigma}}{a_{0}^{2} \sin ^{2} \frac{\mu_{0}}{2} x_{0}+\left|\operatorname{Re} e^{-i \frac{\mu_{0}}{2} x_{0}} \overline{\boldsymbol{a}}\right|^{2}} .
\end{gathered}
$$

Here $a_{0}$ and the complex vector $\boldsymbol{a}$ are defined as

$$
\begin{aligned}
a_{0} & =\cosh r \nu \cosh r_{1} \mu+\hat{\boldsymbol{x}} \cdot \hat{\boldsymbol{y}} \sinh r \nu \sinh r_{1} \mu, \\
\boldsymbol{a} & =\sinh r \nu \cosh r_{1} \mu \hat{\boldsymbol{x}}+\cosh r \nu \sinh r_{1} \mu \hat{\boldsymbol{y}}+i \sinh r \nu \sinh r_{1} \mu \hat{\boldsymbol{x}} \times \hat{\boldsymbol{y}}
\end{aligned}
$$

where $\nu:=\left(\mu_{0} / 2\right)-\mu$ (not a space index), $\hat{\boldsymbol{x}}:=\boldsymbol{x} /|\boldsymbol{x}|$ and $\hat{\boldsymbol{y}}:=\boldsymbol{y} /|\boldsymbol{y}|$ are normalized vectors. Finally, we define $U$ up to $\mathrm{U}(2)$ gauge transformation from the normalization condition

$$
(V, V):=U^{\dagger} U+\int_{I_{1}} v_{1}^{\dagger} v_{1} d s+\int_{I_{2}} v_{2}^{\dagger} v_{2} d s=1_{2},
$$

which reads

$$
U^{\dagger} U+C_{1}^{\dagger} C_{1}+C_{2}^{\dagger} C_{2}=1_{2}, U=\frac{1}{\sqrt{N}} u,
$$


with $u \in \mathrm{U}(2)$. A straightforward calculation shows

$$
N=1+\frac{\lambda^{2}}{2 M}\left\{N_{1}\left(\cosh 2 r \nu-\sinh 2 r \nu(\hat{\boldsymbol{x}})_{3}\right)+N_{2}\left(\cosh 2 r_{1} \mu+\sinh 2 r_{1} \mu(\hat{\boldsymbol{y}})_{3}\right)\right\},
$$

where

$$
M=\cosh 2 r \nu \cosh 2 r_{1} \mu+\sinh 2 r \nu \sinh 2 r_{1} \mu \hat{\boldsymbol{x}} \cdot \hat{\boldsymbol{y}}-\cos \mu_{0} x_{0} .
$$

From this Weyl spinor, we find the gauge potential for $(1,1)$-calorons are

$$
A_{\mu}=i\left\{\sum_{j=1,2}\left(C_{j}^{\dagger} \int_{I_{j}} \tilde{v}_{j}^{\dagger} \partial_{\mu} \tilde{v}_{j} d s C_{j}+C_{j}^{\dagger} \partial_{\mu} C_{j}\right)+\frac{1}{N} u^{\dagger} \partial_{\mu} u+\frac{1}{2} \partial_{\mu} \frac{1}{N} 1_{2}\right\} .
$$

The large distance limit (LDL), $d \rightarrow \infty$ turns out to be

$$
A_{0}=\frac{1}{2}\left(\mu_{0}-3 \mu\right) \sigma_{3}, A_{j}=0,
$$

so that this leads to a pure gauge.

Open Access. This article is distributed under the terms of the Creative Commons Attribution License (CC-BY 4.0), which permits any use, distribution and reproduction in any medium, provided the original author(s) and source are credited.

\section{References}

[1] D.J. Gross, R.D. Pisarski and L.G. Yaffe, $Q C D$ and Instantons at Finite Temperature, Rev. Mod. Phys. 53 (1981) 43 [inSPIRE].

[2] M. Shifman and M. Ünsal, Confinement in Yang-Mills: Elements of a Big Picture, Nucl. Phys. Proc. Suppl. 186 (2009) 235 [arXiv:0810.3861] [INSPIRE].

[3] D. Diakonov, Topology and confinement, Nucl. Phys. Proc. Suppl. 195 (2009) 5 [arXiv: 0906.2456] [INSPIRE].

[4] D. Diakonov, N. Gromov, V. Petrov and S. Slizovskiy, Quantum weights of dyons and of instantons with nontrivial holonomy, Phys. Rev. D 70 (2004) 036003 [hep-th/0404042] [INSPIRE].

[5] E. Poppitz, T. Schäfer and M. Ünsal, Universal mechanism of (semi-classical) deconfinement and theta-dependence for all simple groups, JHEP 03 (2013) 087 [arXiv:1212.1238] [INSPIRE].

[6] D. Diakonov and N. Gromov, $\mathrm{SU}(N)$ caloron measure and its relation to instantons, Phys. Rev. D 72 (2005) 025003 [hep-th/0502132] [INSPIRE].

[7] N. Gromov and S. Slizovskiy, Fermionic determinant for $\mathrm{SU}(N)$ caloron with nontrivial holonomy, Phys. Rev. D 73 (2006) 025022 [hep-th/0507101] [INSPIRE].

[8] S. Slizovskiy, Determinant of the $\mathrm{SU}(N)$ caloron with nontrivial holonomy, Phys. Rev. D 76 (2007) 085019 [arXiv:0707.0851] [INSPIRE].

[9] D. Diakonov and V. Petrov, Confining ensemble of dyons, Phys. Rev. D 76 (2007) 056001 [arXiv: 0704.3181] [INSPIRE]. 
[10] K.-M. Lee and P. Yi, Monopoles and instantons on partially compactified D-branes, Phys. Rev. D 56 (1997) 3711 [hep-th/9702107] [INSPIRE].

[11] F. Bruckmann, D. Nogradi and P. van Baal, Constituent monopoles through the eyes of fermion zero modes, Nucl. Phys. B 666 (2003) 197 [hep-th/0305063] [INSPIRE].

[12] F. Bruckmann, D. Nogradi and P. van Baal, Higher charge calorons with non-trivial holonomy, Nucl. Phys. B 698 (2004) 233 [hep-th/0404210] [inSPIRE].

[13] P. Gerhold, E.M. Ilgenfritz and M. Müller-Preussker, An $\mathrm{SU}(2)$ KvBLL caloron gas model and confinement, Nucl. Phys. B 760 (2007) 1 [hep-ph/0607315] [InSPIRE].

[14] R. Larsen and E. Shuryak, Interacting ensemble of the instanton-dyons and the deconfinement phase transition in the $\mathrm{SU}(2)$ gauge theory, Phys. Rev. D 92 (2015) 094022 [arXiv: 1504.03341] [INSPIRE].

[15] E. Shuryak and T. Sulejmanpasic, Holonomy potential and confinement from a simple model of the gauge topology, Phys. Lett. B 726 (2013) 257 [arXiv:1305.0796] [INSPIRE].

[16] T.C. Kraan and P. van Baal, Periodic instantons with nontrivial holonomy, Nucl. Phys. B 533 (1998) 627 [hep-th/9805168] [INSPIRE].

[17] K.-M. Lee and C.-h. Lu, SU(2) calorons and magnetic monopoles, Phys. Rev. D 58 (1998) 025011 [hep-th/9802108] [INSPIRE].

[18] D. Harland, Large scale and large period limits of symmetric calorons, J. Math. Phys. 48 (2007) 082905 [INSPIRE].

[19] A. Nakamula and J. Sakaguchi, Multi-Calorons Revisited, J. Math. Phys. 51 (2010) 043503 [arXiv: 0909.1601] [INSPIRE].

[20] J. Cork, Symmetric calorons and the rotation map, arXiv:1711.04599 [INSPIRE].

[21] E.B. Bogomolny, Stability of Classical Solutions, Sov. J. Nucl. Phys. 24 (1976) 449 [inSPIRE].

[22] M.K. Prasad and C.M. Sommerfield, An Exact Classical Solution for the 't Hooft Monopole and the Julia-Zee Dyon, Phys. Rev. Lett. 35 (1975) 760 [inSPIRE].

[23] M.F. Atiyah, N.J. Hitchin, V.G. Drinfeld and Yu. I. Manin, Construction of Instantons, Phys. Lett. A 65 (1978) 185 [inSPIRE].

[24] W. Nahm, A Simple Formalism for the BPS Monopole, Phys. Lett. B 90 (1980) 413 [INSPIRE].

[25] W. Nahm, Self-dual monopoles and calorons, Lect. Notes Phys. 201 (1984) 189.

[26] B.J. Harrington and H.K. Shepard, Periodic Euclidean Solutions and the Finite Temperature Yang-Mills Gas, Phys. Rev. D 17 (1978) 2122 [InSPIRE].

[27] B.J. Harrington and H.K. Shepard, Thermodynamics of the Yang-Mills Gas, Phys. Rev. D 18 (1978) 2990 [INSPIRE].

[28] R. Jackiw, C. Nohl and C. Rebbi, Conformal Properties of Pseudoparticle Configurations, Phys. Rev. D 15 (1977) 1642 [INSPIRE].

[29] P. Rossi, Propagation Functions in the Field of a Monopole, Nucl. Phys. B 149 (1979) 170 [INSPIRE].

[30] R.S. Ward, Symmetric calorons, Phys. Lett. B 582 (2004) 203 [hep-th/0312180] [InSPIRE]. 
[31] D. Muranaka, N. Sawado, A. Nakamula and K. Toda, Numerical Nahm transform for 2-caloron solutions, Phys. Lett. B 703 (2011) 498 [arXiv:1105.2092] [INSPIRE].

[32] S.A. Brown, H. Panagopoulos and M.K. Prasad, Two Separated SU(2) Yang-Mills Higgs Monopoles in the Adhmn Construction, Phys. Rev. D 26 (1982) 854 [inSPIRE].

[33] A. Chakrabarti, Periodic Generalizations of Static, Selfdual SU(2) Gauge Fields, Phys. Rev. D 35 (1987) 696 [INSPIRE].

[34] A. Chakrabarti, Quasiperiodic instantons, Phys. Rev. D 38 (1988) 3219 [InSPIRE].

[35] X. Chen and E.J. Weinberg, ADHMN boundary conditions from removing monopoles, Phys. Rev. D 67 (2003) 065020 [hep-th/0212328] [INSPIRE].

[36] G. Etesi and M. Jardim, Moduli spaces of self-dual connections over asymptotically locally flat gravitational instantons, Commun. Math. Phys. 280 (2008) 285 [math/0608597] [INSPIRE]. 